\title{
1 Human SYNGAP1 Regulates the Development of Neuronal Activity by \\ Controlling Dendritic and Synaptic Maturation
}

\author{
Nerea Llamosas ${ }^{1}$, Vineet Arora ${ }^{1}$, Ridhima Vij ${ }^{2,3}$, Murat Kilinc ${ }^{1}$, Lukasz Bijoch $^{4}$, Camilo \\ Rojas $^{1}$, Adrian Reich ${ }^{5}$, BanuPriya Sridharan ${ }^{6 \infty}$, Erik Willems ${ }^{7}$, David R. Piper ${ }^{7}$, Louis \\ Scampavia ${ }^{6}$, Timothy P. Spicer ${ }^{6}$, Courtney A. Miller ${ }^{1,6}$, J. Lloyd Holder Jr²,3, Gavin \\ Rumbaugh $^{1^{*}}$
}

8

91 Department of Neuroscience, Scripps Research, Jupiter, Florida 33458, USA

10 2Jan and Dan Duncan Neurological Research Institute at Texas Children's Hospital, Houston

11 Texas 77030 , USA

$12{ }^{3}$ Department of Pediatrics, Baylor College of Medicine, Houston, Texas 77030, USA

$13{ }^{4}$ Laboratory of Neurobiology, BRAINCITY, Nencki Institute of Experimental Biology, Polish

14 Academy of Sciences, Warsaw, Poland

$15{ }^{5}$ Center for Computational Biology and Bioinformatics, Scripps Research, Jupiter, Florida 33458, 16 USA

$17{ }^{6}$ Department of Molecular Medicine, Scripps Research, Jupiter, Florida 33458, USA

$18 \quad{ }^{7}$ Cell Biology, Thermo Fisher Scientific, Carlsbad, California 92008, USA œCurrent Address: GSK 1250 S Collegeville Rd., Collegeville, PA 19426,

${ }^{*}$ Corresponding Author:

23 Gavin Rumbaugh

24 Department of Neuroscience

25130 Scripps Way \#3B3

26 Jupiter, FL 33458

27 gavin@scripps.edu 


\section{Abstract}

SYNGAP1 is a major genetic risk factor for global developmental delay, autism spectrum

32 disorder, and epileptic encephalopathy. De novo loss-of-function variants in this gene cause a

33 neurodevelopmental disorder defined by cognitive impairment, social-communication disorder,

34 and early-onset seizures. Cell biological studies in mouse and rat neurons have shown that

35 Syngap1 regulates developing excitatory synapse structure and function, with loss-of-function

36 variants driving formation of larger dendritic spines and stronger glutamatergic transmission.

37 However, studies to date have been limited to mouse and rat neurons. Therefore, it remains

38 unknown how SYNGAP1 loss-of-function impacts the development and function of human neurons. To address this, we employed CRISPR/Cas9 technology to ablate SYNGAP1 protein expression in neurons derived from a human induced pluripotent stem cell line (hiPSC).

41 Reducing SynGAP protein expression in developing hiPSC-derived neurons enhanced dendritic

42 morphogenesis, leading to larger neurons compared to those derived from isogenic controls.

43 Consistent with larger dendritic fields, we also observed a greater number of morphologically

44 defined excitatory synapses in cultures containing these neurons. Moreover, neurons with

45 reduced SynGAP protein had stronger excitatory synapses and expressed synaptic activity

46 earlier in development. Finally, distributed network spiking activity appeared earlier, was

47 substantially elevated, and exhibited greater bursting behavior in SYNGAP1 null neurons. We conclude that SYNGAP1 regulates the postmitotic maturation of human neurons made from hiPSCs, which influences how activity develops within nascent neural networks. Alterations to

50 this fundamental neurodevelopmental process may contribute to the etiology of SYNGAP1-

51 related disorders. 


\section{Introduction}

Pathogenic loss-of-function variants in the SYNGAP1 gene are causally-linked to a range of neuropsychiatric disorders, including global developmental delay (GDD)/intellectual disability (ID) (Hamdan et al., 2009; Rauch et al., 2012; Deciphering Developmental Disorders, 2015, 2017) and severe epilepsy (Carvill et al., 2013; von Stulpnagel et al., 2015; Vlaskamp et al., 2019). SYNGAP1 is also strongly implicated in autism spectrum disorders (Rauch et al., 2012; O'Roak et al., 2014) and was recently identified as one of three genes that impart the highest risk for developing autistic features (Satterstrom et al., 2020). While pathogenic variants in SYNAGP1 are overall rare, they are common relative to the pool of genes capable of causing sporadic neurodevelopmental disorders, explaining up to $\sim 1 \%$ of GDD/ID cases (Berryer et al., 2013; Parker et al., 2015). While the exact incidence of SYNGAP1 pathogenicity remains unknown, early estimates are 1/10,000 (Parker et al., 2015; Weldon et al., 2018), which is in the range of other monogenic disorders that are more extensively studied by the scientific community. Causality of SYNGAP1 pathogenicity is now established because there are no known loss-of-function variants in $>141,000$ neurotypical individuals from the gnomAD database and all known patients with clearly pathogenic variants are diagnosed with a neurodevelopmental disorder (Mignot et al., 2016). Moreover, the pLI ratio for SYNGAP1 is 1 (Lek et al., 2016; Jimenez-Gomez et al., 2019), demonstrating that the human gene is extremely intolerant of loss-of-function variants. Based on substantial clinical evidence, proper SYNGAP1 expression is required for normal human brain development and function.

Syngap1 gene function has been studied in rodent neurons (Kilinc et al., 2018; Gamache et al., 2020). Syngap1 is a potent regulator of dynamic processes required for Hebbian plasticity at excitatory synapses. Heterozygous knockout mice exhibit deficits in hippocampal LTP evoked through a variety of synaptic stimulation protocols (Komiyama et al., 2002; Kim et al., 2003). This function of SynGAP protein is consistent with cognitive impairment commonly observed in SYNGAP1 patients because Hebbian plasticity at excitatory synapses is thought to contribute importantly to learning. Genetic re-expression of Syngap1 in adult mutant mice rescues hippocampal LTP and associated downstream signaling pathways (Ozkan et al., 2014). Thus, SynGAP regulation of synapse plasticity is a dynamic function of the protein that is retained throughout life. Hundreds of genes regulate synaptic plasticity as referenced by the Gene

85 Ontology Browser (366 genes;

86 http://www.informatics.jax.org/vocab/gene ontology/GO:0048167). However, most of them do 87 not cause disease when heterozygously expressed, as is the case for SYNGAP1 (Carvill et al., 
2013; Deciphering Developmental Disorders, 2015, 2017; Satterstrom et al., 2020). Therefore, SYNGAP1 likely has additional functions beyond regulation of synapse plasticity that contribute to disease etiology. Indeed, there are additional reported functions of the Syngap1 gene. SynGAP expression in developing mouse neurons acts to regulate the maturation rate of excitatory synapse strength and this function is independent from its role in plasticity. SynGAP protein expression rises quickly during postnatal development (Gou et al., 2020) and its expression during this period is critical for shaping the strength of nascent excitatory synapses (Clement et al., 2012; Clement et al., 2013). Syngap1 heterozygous mice have enhanced excitatory synapse function in the developing cortex and hippocampus, which is thought to contribute to early onset of behavioral deficits and seizures observed in these animals. In contrast to Hebbian processes, this function of rodent Syngap1 is linked to biological process unique to developing neurons. Enhanced baseline excitatory synaptic strength in hippocampal neurons is transiently observed during the first three postnatal weeks of brain development and inducing heterozygosity of Syngap1 beyond this period has minimal effect on resting synaptic function in these neurons (Clement et al., 2012).

103

The understanding of how this gene contributes to disease-relevant biology is limited because information on its function in human neurons is lacking. This is limiting because there are fundamental differences in how human and rodent brains develop. For example, humans express neoteny, or slowing of development, which is thought to promote an extended period of neural network refinement that promotes higher cognitive functions. An example of neoteny at the neurobiological level is the relative pace of human neuron development compared to rodents (Petanjek et al., 2011; Charrier et al., 2012), with human neurons exhibiting a much slower pace of postmitotic differentiation. Given that Syngap1 alters measures of neuronal maturation in rodents (Clement et al., 2012; Clement et al., 2013; Aceti et al., 2015), this

113 function of the gene may be amplified in slower developing human neurons. To test this idea,

114 we created SYNGAP1 knockout human induced pluripotent stem cell (hiPSC) lines using

115 CRISPR/Cas9 technology. These iPSCs were then differentiated into neurons (iNeurons) and

116 cultures were assessed for various parameters of neuronal maturation. We found that human

117 iNeurons lacking SynGAP expression exhibited accelerated dendritic morphogenesis, increased

118 accumulation of postsynaptic markers, early expression of synapse activity, enhanced excitatory

119 synaptic strength, and early onset of neural network activity. We conclude that SYNGAP1

120 regulates the postmitotic differentiation rate of developing human neurons and disrupting this

121 process impacts the function of nascent neural networks. These observations in human neurons 
122 are consistent with findings from rodent studies, indicating that control of neuronal maturation is

123 a species-conserved function of the gene. Therefore, disruptions to this fundamental

124 neurodevelopmental process may contribute to the etiology of SYNGAP1-related brain

125 disorders.

126

127 Material and Methods

128 Maintenance of hiPSC cultures

129 All hiPSC work was performed in accordance with approved protocols from appropriate

130 Institutional Review Boards. All products were purchased from Thermo Fisher Scientific unless

131 otherwise noted. The stable human episomal Cas9 hiPSC cell line was obtained from Thermo

132 Fisher Scientific (A33124) and was expanded according to the manufacturer's suggested

133 protocol. This line was previously used for generating neurons (Sridharan et al., 2019). Briefly,

134 culture plates were coated with Vitronectin-N (A14700), diluted 1:100 in DPBS (14190094), and

135 incubated at $37^{\circ} \mathrm{C}$ for at least $1 \mathrm{~h}$ prior to iPSC plating. Cryopreserved iPSC cells were gently

136 thawed in a $37^{\circ} \mathrm{C}$ water bath and transferred to a $15 \mathrm{~mL}$ conical tube with Complete iPSC

137 Medium $+1 \%$ RevitaCell supplement (A2644501). Cells were then centrifuged at $200 \times \mathrm{g}$ for

1384 min and the iPSC pellet was re-suspended in fresh medium and plated on vitronectin coated

139 flasks. Twenty-four hours later, cells were switched and maintained in Complete Stemflex

140 Medium (w/o RevitaCell) with daily medium changes until $70 \%$ confluent. Cells were then

141 harvested with TrypLE Select (12563011) and further maintained or plated for experimental

142 purposes. For limiting dilution cloning, iPSCs were plated in 96-well plates coated with $2.5 \mu \mathrm{m} / \mathrm{ml}$

143 rhLaminin-521 (A29248).

144

145 Generation of SYNGAP1 KO hiPSC lines

146 Guide RNA ( $g$ RNA) sequences targeting exon 7 of SYNGAP1 were selected using the Zhang

147 lab CRISPR design tool (http://zlab.bio/guidedesign-resources) and acquired from IDT in single

148 guide RNAs (sgRNA) format. Cas9-iPSCs were transfected with sgRNAs by using

149 Lipofectamine CRISPRMAX (Thermo Scientific,CMAX00001) according to manufacturer's

150 instructions. Editing efficiency of individual sgRNAs was determined using GeneArt Genomic

151 Cleavage Detection Kit (Thermo Scientific, A24372). sgRNA-5 (target sequence 5'-

152 TCTTTCGGCCGCAGACCGAC-3') demonstrated the highest efficiency and was selected for

153 downstream applications. To generate the SYNGAP1 KO iPSC lines, cells were transfected with

154 sgRNA-5. Twenty-four hours after the transfection, cells were plated in rhLaminin-521 coated

155 96-well plates with an average density of 0.5 cells/well. Colonies derived from a single cell were 
expanded and cryopreserved with Recovery cell culture freezing medium (Thermo Scientific, 12648010). Approximately 70 colonies originating from a single cell were analyzed for Indels around the sgRNA targeting site. Multiple clones with either unedited (WT) or edited (potential $\mathrm{KO})$ sequences were isolated and expanded. Potential KO clones with "clean" Sanger sequence traces were prioritized. Pluripotency of individual clones were confirmed via TaqMan Array Human Stem Cell Pluripotency Panel (4385344) according to manufacturers' instructions. Each expanded clone was tested, and confirmed negative, for mycoplasma contamination using Universal Mycoplasma Detection Kit (ATCC, 30-1012K).

\section{Whole exome sequencing (WES)}

Genomic DNA from the four experimental clones and a sample of the original Cas9 iPSC line (before CRISPR transfection) were extracted using PureLink Genomic DNA mini Kit (Invitrogen \#k1820-02) using included instructions. Genomic DNA from each of the five samples was shipped to HudsonAlpha Institute for Biotechnology, Genome Sequencing Center (Huntsvile, $A L)$ for WES.

\section{Library Preparation and Quality Control}

DNA samples were normalized to 1,000 ng of DNA in $50 \mu$ of water. Following normalization, samples were acoustically sheared via Covaris LE-220 instrument to a final fragment size of 350-400bp. The sheared DNA was then transformed into a standard Illumina paired-end sequencing library via standard methods. The sheared DNA was end-repaired and A-tailed using Roche-Kapa End-Repair and A-Tailing kits under the manufacturer's recommended conditions. Standard Illumina paired-end adaptors were ligated to the A-tailed DNA. Following ligation, the reactions were purified using AMPure XP beads. The purified ligated DNA was amplified via PCR using Roche KAPA HIFI PCR reagents using 4 cycles of PCR. The primers used in the PCR step introduced 8-base, unique, dual indexes in the i5 and i7 positions to allow sample identification/demultiplexing following sequencing. The final library was quality controlled using size verification via PerkinElmer LabChip GX and real-time PCR using the Roche KAPA SYBR FAST qPCR Master Mix, primers and standards according to the manufacturer's directions. Libraries were normalized to $1.4 \mathrm{nM}$ stocks for use in clustering and sequencing.

\section{IDT Exome Capture and Quality Control}

Post-library construction, samples were multiplexed for capture at 5 samples per pool with each sample contributing a maximum of $300 \mathrm{ng}$ or a minimum of $200 \mathrm{ng}$ to each pool. Pooled samples 
were purified with beads and eluted in a volume of $30 \mu \mathrm{l}$. Pooled samples were hybridized with the NimbleGen SeqCap EZ Exome v3 probes with minor modifications for automation. Briefly, multiplexed samples were dried down in the presence of COT-1 and a blocker mix for $1.5 \mathrm{hrs}$. Libraries were then resuspended in a mix of hybridization buffer and baits. Libraries were hybridized overnight at $65^{\circ} \mathrm{C}(72 \mathrm{hrs})$. Post-hybridization takedown occurred 72 hours later. Briefly, captured libraries were bound to streptavidin beads. Once bound, washing occurred per manufacturer's recommendations. Final elution of captured libraries was in $20 \mu$ l of nucleasefree water. Libraries were amplified with six cycles of PCR and a final bead purification. Posthybridization exome concentrations were measured via Picogreen and library sizes were determined via the LabChip GX Touch HT (PerkinEImer). Additionally, libraries were quantitated with real-time PCR using the KAPA Library Quantification Kit (Roche) per manufacturer's instructions to determine final library nanomolarity. Final exome libraries were pooled at a concentration of $1.8 \mathrm{nM}$. The pooled exome libraries were distributed across four lanes on an S4 flow cell and sequenced using 150 base pair paired-end approach on a NovaSeq 6000 instrument (Illumina). All sequencing was performed on the Illumina NovaSeq 6000 platform by loading a pool samples to the equivalent loading of 24 samples per flowcell. Following sequencing all basecalling was performed using standard Illumina software to generate the final FASTQ files for each sample. Alignment and variant calling was performed via the Edico/lllumina DRAGEN pipeline to verify coverage and performance. Samples yielded a minimum of $440 \mathrm{M}$ paired reads at 150 nt read length with a mean coverage of greater than $30 \mathrm{X}$.

\section{Karyotyping}

Karyotyping was performed as previously described (Sridharan et al., 2019). Briefly, differentiated iNeurons were assessed for any chromosomal aberrations using the Karyostat $^{\mathrm{TM}}$ assay (Thermo Fisher).

\section{Generation of induced Neurons (iNeurons) from Cas9-iPSC single cell clones}

Ngn2 transcription factor induced iNeurons were generated as previously described (Sridharan et al., 2019) with minor modifications. Briefly, Cas9-hiPSCs were harvested using TrypLE Select and 2 million cells were plated on vitronectin coated T75 flask on day 1. On day 2, medium was removed, and an appropriate amount of lentivirus expressing Ngn2 (Addgene, 52047) and rtTA (Addgene, 20342) were administered in Complete Stemflex Medium including 1\% RevitaCell (MOI 2 for both lentivirus). After $24 \mathrm{~h}$, the medium was aspirated and replaced with Induction Media induce TetO gene expression. The next day, medium was refreshed with Induction Media 
$+2 \mu \mathrm{g} / \mathrm{mL}$ Puromycin (A1113803) which was included for selection of iNs. Twenty-four hours later, iNeurons were harvested using Accutase (A1110501) and plated on PDL coated plates in iNeuron Maintenance Media (Neurobasal (211103049) + 1\% GlutaMax (35050061) + 2\% B27 $(17504044)+10 \mathrm{ng} / \mathrm{ml} \mathrm{BDNF}(\mathrm{PHC7074})+10 \mathrm{ng} / \mathrm{ml}$ GDNF $(\mathrm{PHC7036})+10 \mathrm{ng} / \mathrm{ml}$ NT3 $($ PHC7045) + 2.5\% FBS (10082139), all from Thermo) + $10 \mu \mathrm{g} / \mathrm{ml}$ FuDR (Sigma, F0503) along with primary rat glia (neuron/glia ratio 2.5/1). Half of the medium was changed with fresh iNeuron Maintenance Media every 4-5 days.

\section{Dendritic Tracing}

Each well of a 96-well imaging plate contained $\sim 50,000$ cells per well, consisting of $\sim 32,000$ human induced Neurons (iNs) + 18,000 Primary Rat astrocytes along with $0.1 \%$ ( 50 per well) of EGFP positive human induced Neurons derived from the same clone. eGFP-positive iNeurons were created through a separate induction as stated above, except that an additional lentivirus expressing eGFP under the control of a TET-responsive promoter was included (Addgene Cat \# 30130). eGFP-positive neurons were mixed with eGFP-negative neurons in the 96-well plates. iNeurons derived from either of the WT or KO clones were compared by tracing primary (originating from the soma), secondary, and tertiary dendrites, as well as total dendrite length. Tracing data was obtained by imaging live iNeurons at DIV45 with an InCell Analyzer 6000 automated confocal microscope (20X magnification). A sample of 30 randomly selected neurons per genotype ( $n=3$ per well $\times 10$ wells in a 96-well plate) was selected and then dendrites were traced with the Simple Neurite Tracer (SNT) software plugin distributed by FijiImageJ. Data represents the average lengths in microns for all subtypes of dendrites.

\section{Immunocytochemistry}

iNeurons were re-plated, along with primary rat astrocytes, at a density of iNeurons 200,000/120,000 astrocytes per well, on 15mm cover glass coated with PDL/Fibronectin (Neuvitro, GG-15-Fibronectin), in 24-well plates. At DIV30-45, cells were fixed and labelled with primary antibodies: anti-PSD95 (mouse-raised; Abcam Cat\# ab2723-100ug), anti-GluA1 (Rabbit-raised; Cell Signaling Technology Cat\# 13185S) and anti-MAP2 (Guinea pig-raised; Synaptic Systems Cat \# 188004). Then, secondary antibodies were applied (Goat anti-mouse Alexa 488, Abcam Cat\# ab150113-500ug, Goat anti-rabbit Alexa 568, ThermoFisher Cat\# A11036, Goat anti-guinea pig Alexa 647 ThermoFisher Cat\# A21450). Images of neurons from multiple coverslips per culture were taken under UPlanSApo 100× 1.4 NA oil-immersion objective mounted on Olympus FV1000 laser-scanning confocal microscope (1 image = 1 field- 
of-view). Neuronal somas from individual fields-of-view were manually calculated based on raw MAP2-signals. Total area of MAP2/field-of-view was determined on the area of mask of MAP2 signal. Number of detected particles of GLUA1 and PSD95 per field-of-view was determined based on threshold-based signal masks. Thresholds were kept constant across all images.

\section{Immunoblotting}

iNeurons were co-cultured with rat glia (500,000 induced neurons, 100,000 glia) seeded on 12well plates. After 30-60 days in culture, media was removed and the wells were washed with PBS, after which the PBS was replaced with 200ul of RIPA buffer (Cell Signaling Technology, Danvers, MA) containing Phosphatase Inhibitor Cocktails 2 and 3 (Sigma-Aldrich, St. Louis, MO) and MiniComplete Protease Inhibitor Cocktail (Roche Diagnostics), the wells were scraped using a sterile cell scraper on each well and transferred to tubes in dry ice, and stored at $-80^{\circ} \mathrm{C}$. After thawing on ice, samples were sonicated using a probe sonicator 5 times with 2 sec pulses. Sample protein levels were measured (Pierce BCA Protein Assay Kit, Thermo Scientific, Rockford, IL), and volumes were adjusted to normalize microgram per microliter protein content. $10 \mu \mathrm{g}$ of protein per sample were loaded and separated using SDS-PAGE on 4-15\% gradient stain-free tris-glycine gels (Mini Protean TGX, BioRad, Hercules, CA), transferred to low fluorescence PVDF membranes $(45 \mu \mathrm{m})$ with the Power Blotter- Semi-dry Transfer System (ThermoFisher Scientific). Membranes were blocked with 5\% powdered milk in buffer and probed with pan-SynGAP (1:1,000, \#5539, Cell Signaling) or SynGAP-a2 (abcam, ab77235), overnight at $4^{\circ} \mathrm{C}$ and HRP-conjugated anti-rabbit antibody (1:2,000, W4011, Promega) for $1 \mathrm{hr}$ at room temperature followed by ECL signal amplification and chemiluminescence detection (SuperSignal West Pico Chemiluminescent Substrate; Thermo Scientific, Rockford, IL). Blot band densities were obtained using the ChemiDoc imaging system (BioRad). SynGAP levels of immunoreactivity were assessed by densitometric analysis of generated images with ImageJ. Values were normalized to total protein levels obtained from blots prior to antibody incubations.

\section{Whole Cell Electrophysiology}

iNeuron measurements were performed up to DIV50 as described with minor modifications (Sridharan et al., 2019). For current studies, iNeurons were co-cultured with cryo-recovered primary rat astrocytes (seeded at 20,000 iNs $+10,000$ astrocytes per well) in 24-well plate on $15 \mathrm{~mm}$ coverslips. The co-cultures were maintained in plating medium and additionally supplemented with $10 \mu \mathrm{g} / \mathrm{mL}$ FUDR (cat. no. F0503, Sigma). For whole cell recordings, intrinsic electrical properties were inspected immediately after gaining access to the cell and miniature 
excitatory synaptic currents were recorded in the presence of TTX $(0.5 \mu \mathrm{m})$ at room temperature in voltage-clamp configuration (cells were held at $-60 \mathrm{mV}$ with a Multiclamp $700 \mathrm{~B}$ amplifier, Molecular Devices). The bath solution contained (in mM): $140 \mathrm{NaCl}, 5 \mathrm{KCl}, 2 \mathrm{CaCl}_{2}, 2 \mathrm{MgCl}_{2}, 10$ HEPES-NaOH and 10 Glucose ( $\mathrm{pH}$ to 7.4 adjusted with $\mathrm{NaOH}$ ). Pipettes pulled from borosilicate glass capillary tubes (cat. no. G85150T-4, Warner Instruments) using a P-97 pipette puller (Sutter Instrument) were filled with the following intracellular solution (in $\mathrm{mM}$ ): $123 \mathrm{~K}$ gluconate, $10 \mathrm{KCl}, 1 \mathrm{MgCl}_{2}, 10 \mathrm{HEPES}-\mathrm{KOH}, 1 \mathrm{EGTA}, 0.1 \mathrm{CaCl}_{2}, 1 \mathrm{~K}_{2}-\mathrm{ATP}, 0.2 \mathrm{Na}_{4}$-GTP and 4 glucose ( $\mathrm{pH}$ adjusted to 7.4 with $\mathrm{KOH}$ ). Resistance of the pipettes filled with the intracellular solution was between 3-5 $\mathrm{m} \Omega$. Series resistance was monitored without compensation with $5 \mathrm{mV}$ depolarizing steps (200 ms) induced every 60 s to ensure sufficient and stable electrical access to the cell. Data were sampled at $10 \mathrm{kHz}$, post hoc filtered and analyzed offline using Clampfit (Molecular Devices). Single peak mEPSCs were detected using a semiautomated sliding template detection procedure in which the template was generated by averaging multiple spontaneous currents. Each detected event was visually inspected and discarded if the amplitude was $<7 \mathrm{pA}$.

\section{Microelectrode Array (MEA) Analysis}

\section{Cell culture and NPC differentiation}

Individual SYNGAP1 WT and KO iPSC clones were maintained on Matrigel-coated plates in Stem Flex media (Fisher Scientific). Neural Progenitor Cells (NPCs) were differentiated from iPSCs using a dual SMAD inhibition protocol (Jiang et al., 2017). Briefly, stem cell lines were dissociated using Accutase and embryoid bodies were generated from the stem cell lines in the Aggrewells using Neural Proliferation medium (NPM) along with BMP and WNT inhibitors (Dorsomorphin: DM; 4mM and SB-431542:SB 10mM; Sigma Aldrich), administered on Day 2 of neural induction. At $\sim$ Day 5, EBs were gently collected and plated on Matrigel coated plates for the formation of rosettes. To promote dorsalization, 10mM Cyclopamine (CCP; Stem Cell technologies) was added to the plates starting Day 6. Both inhibitors and CCP were added to the media until Day 9. Rosettes were collected between Day 14 and 16 and plated on gelatincoated plates so that the non-neural cells were preferentially removed from floating neural progenitors, which were then dissociated to form a monolayer culture of neural progenitor cells. NPCs were grown and expanded on Matrigel coated plates before the cells were plated directly on a MEA plate for neuronal differentiation.

\section{MEA analysis and neuronal differentiation}


We employed an MEA system (Axion Biosystems) to perform neurophysiological characterization of iNeurons. Neuronal differentiation of NPCs was performed directly on MEA plates. $1.6 \times 10^{4}$ NPCs suspended in a $5 \mu$ ldroplet of NPM (neural precursor medium) were plated as on top of a 16-electrode array (area $\sim 1 \mathrm{~mm}^{2}$ ) inside a single well of 48 -well MEA plate pre-treated with $0.1 \% \mathrm{PEI}$ solution prepared in borate buffer $(\mathrm{pH}=8.4)$. Two days later, neuronal differentiation was initiated using in neuronal induction medium (NIM, prepared from equal volumes of DMEM/F12 and neurobasal medium without growth factors) prepared in-house. NIM was exchanged every other day for 7 days. Differentiation of the NPCs into forebrain cortical neurons was performed using previously established neuronal differentiation medium, NDM, which includes a cocktail of differentiation factors (BDNF, GDNF, NT-3, dibutyryl-cAMP, ascorbic acid) (Jiang et al, 2017 Nature Comm). Post-differentiation, NDM was replaced with BrainPhys for further maturation (Stem Cell technologies), and neurons were cultured for at least one week before neuronal activity was recorded. Neuronal activity was recorded continuously for 5 minutes from the multi-well MEA plate each week until 6 weeks of neuronal maturation, post-differentiation. Field potential changes were recorded and analyzed using Axis Navigator and Axis metric plotting software (Axion Biosystems). Temporal raster plots were generated using Neural Metric Tool software (Axion Biosystems). For data analysis, a burst was identified as a group of at least 5 spikes, separated by an inter-spike interval (ISI) of less than 100 milliseconds. Network bursts were defined as a minimum of 50 spikes with a maximum ISI of $100 \mathrm{~ms}$ covering at least $35 \%$ of electrodes in a well.

\section{Statistics}

GraphPad Prism 8 software was used for all statistical analysis. All data were tested for normality. Accordingly, parametric or non-parametric tests were applied. For tracing data analyses, clonal comparisons were performed using Kruskal-Wallis test followed by Dunn's multiple comparison test. For genotype comparisons Mann-Whitney tests were applied. For immunostaining experiments, Mann-Whitney tests or unpaired two-tailed t-tests were used. For clonal comparisons of electrophysiological data Kruskal-Wallis followed by corrected Dunn's multiple comparison tests or One-way ANOVA followed by Tukey tests were used. Statistical differences of percentage mEPSC-expressing neurons were determined by Fischer exact test pair-wise comparisons. For genotypic comparisons of whole cell electrophysiological data, Mann-Whitney $U$ tests or Unpaired t-tests were performed. When comparing cumulative probability data between clones or genotypes the Kolmogorov-Smirnov test was used. For multielectrode array studies, statistical analyses among clones was performed using two-way 
RM ANOVA followed by Tukey's multiple comparison test and for genotype comparisons twoway RM ANOVA followed by Bonferroni's multiple comparison. Data throughout the text are presented either as box-and-whisker plots where the center, boxes and whiskers represent the median, interquartile range, and min to max, respectively, or as mean \pm SEM. Differences were considered to be significant for $p<0.05$. Exact $p$-values are reported when provided by the software.

\section{Results}

To create SYNGAP1 null hiPSCs, we performed CRISPR editing of a common exon within the human locus. Exon 7 was targeted (Fig. 1A) for non-homologous end joining (NHEJ) repair for the following reasons: 1) it is a common exon present in most, if not all, SYNGAP1 transcripts (McMahon et al., 2012; Gou et al., 2020); 2) it is downstream of multiple stop-gain or small indel patient-specific variants (Jimenez-Gomez et al., 2019); 3) targeting it in other species results in ablation of SynGAP protein (Kim et al., 2003; Clement et al., 2012). Four single cell clones were identified and selected for downstream analysis. These clones contained either an edited (KO Clone \#4 and \#38) or unedited (WT- Clone \#6 and \#30) Exon 7 (Fig. 1A-B). Sanger sequencing of the putative KO clones contained "clean" sequence both up and downstream of the Cas 9 cut site. Importantly, karyotyping analysis (Fig. 1C) revealed no large alterations to chromosomal structure in any of the four clones. Moreover, each of the clones passed self-renewal and pluripotency checks and tested negative for mycoplasma contamination. The type of variants present, combined with the Sanger sequence traces, suggested that both clones contained biallelic indel frameshift variants, which would be expected to cause nonsense mediated decay of SYNGAP1 transcripts and disruption to SynGAP protein. To test this prediction, glutamatergic neurons were produced from each of the four clones using the Ngn2 induction method (Zhang et al., 2013). After $\sim 30-60$ days of neuronal development, samples were immunoblotted for SynGAP protein levels. As predicted, neurons derived from both "KO" clones had significantly lower levels of SynGAP protein than "WT" clones. Reduced SynGAP signal was observed with antibodies recognizing either a core region of the protein (Pan-SynGAP), or to the C-terminus of a specific splice variant ( $\alpha 2$; Fig. 1D-E). Given that SynGAP signal is $\sim 10 \%$ of control levels, the two KO clones appeared to produce iNeurons with nominal SynGAP protein expression.

We next performed whole exome sequencing (WES) to quantify the genetic differences among the clones. In general, the four clones had very little genetic drift across the protein coding portion of the genome. We observed only a few high confidence exonic small indels in each of 
the four CRISPR clones (Table 1). None of these indels were shared within the same gene and none of them appeared to be homozygous, except for the two unique indels identified in Exon7 of SYNAGP1 (Fig. 1F). Thus, unbiased read-mapping of WES identified the sequences used to select the two "KO" clones and these sequences appeared to be the most significant deviations amongst the four clones (Fig. 1A-B; Table 1). Therefore, these four clones are essentially isogenic, with the exception of the homozygous disruptive variants present in SYNGAP1. We next performed in-depth mapping of SYNGAP1 exons to further characterize potential off-target effects of Cas9 genome editing (Fig. 1G). Comparing normalized reads of each of the four clones relative to the original iPSC line revealed that SYNGAP1 exon structure was largely intact. However, we did observe a $\sim 50 \%$ reduction in mapped reads in the targeted exon of KO clone \#38. Flanking exons had normal read depths (Fig. 1H). This was suggestive of a large deletion that encompassed exon $6 / 7$, but was less than $4 \mathrm{~Kb}$ in size. Genomic PCR failed to detect a band shift (Fig. 1I), though PCR amplification in this region was limited to $\sim 2.2 \mathrm{~Kb}$. Thus, for clone \#38, there were likely two distinct Indels in each of the SYNGAP1 copies. Copy 1 contained an 8bp deletion (Fig. 1B; Table 1), while the other copy likely contained an undefined indel $(<4 \mathrm{~Kb})$ that prevented amplification by traditional PCR. In contrast, clone \#4 appeared to contain a bi-allelic single base deletion in exon 7. In each case, these indels produced nominal SynGAP protein in induced neurons (Fig. 1D-E). We conclude that the two "KO" clones, when paired with the isogenic "WT" clones, were suitable to determine the impact of SynGAP loss-of-function on human neuron development and function.

Syngap1 loss-of-function in rodent neurons disrupts the maturation rate of dendrites and synapses. Therefore, we examined dendritic morphogenesis in developing iNeurons produced from each of the four human iPSC clones. Dendritic morphology was measured at day in vitro (DIV) 45 by tracing dendrites of sparsely labeled eGFP-positive iNeurons (Fig. 2A). Relative to each isogenic control line, total dendritic fields were substantially larger in iNeurons derived from SYNGAP1-KO clones. This difference was observed at the level of individual clones (Fig. 2A-B) and when clones were grouped by genotype (Fig. 2B). Examination of the length by dendritic category (e.g. primary) revealed that, compared to WT clones, KO clones generally had longer primary and secondary dendrites (Fig. 2A, C-E). The lack of a clonal difference within tertiary dendrites likely reflected a lower statistical power, as many neurons lacked these structures. In contrast to length, the complexity of dendritic arbors was unaffected by SYNGAP1 disruption. Clonal and genotype effects of SYNGAP1 were not observed for total dendrites (Fig. 2F). Moreover, no SYNGAP1 effects were observed for each dendrite subtype (Fig. 2G-I). 
Tracing studies suggested that reduced SynGAP expression leads to iNeurons with larger dendritic fields. To confirm this, we performed an orthogonal analysis, consisting of immunocytochemical labeling of dendritic and synaptic proteins, in neurons derived from one

432 pair of isogenic WT or KO iPSCs (Fig. 3A-B). The MAP2 area was enhanced in KO cultures

433 (Fig. 3A-C) and was not due to more KO neurons plated in these cultures (Fig. 3A-E). Cultured neurons with longer dendrites would be expected to have an increase in absolute numbers of postsynaptic structures. Indeed, absolute numbers of PSD95 and GLUA1 structures were also increased in the KO culture (Fig. 3A-C). The effect of genotype on synaptic labeling was still significant, albeit with a much smaller effect size, when PSD95 and GLUA1 structures were normalized to MAP2 area (Fig. 3F). These labeling studies support the idea that disrupting SynGAP expression results in cultures comprised of larger neurons with more postsynaptic structures.

The observation of larger iNeurons with increased numbers of postsynaptic structures prompted us to investigate the functional maturation of iNeurons with reduced SynGAP protein expression. Intrinsic membrane properties and the onset of glutamatergic synaptic activity are two measures that are developmentally regulated in Ngn2-induced neurons (Zhang et al., 2013). To test the idea that reducing SynGAP expression alters the maturation of iNeurons, we performed whole-cell voltage-clamp recordings at two developmental time points (DIV20-30 and DIV40-50; Fig. 4A-B). At DIV20-30, intrinsic membrane properties of all clones were characteristic of immature neurons (i.e. relatively low capacitance and high input resistance;

450 Fig. 4C-D). We did not observe clonal or genotype differences in resting membrane potential, capacitance, or resistance at this time point (Fig. 4C-E). However, we did observe that neurons

452 made from SYNGAP1-KO hiPSCs showed earlier synaptic activity during development.

453 Although some iNeurons from all clones exhibited miniature excitatory postsynaptic currents

454 (mEPSCs) at this time point (Fig. 4F), the proportion of $m$ EPSC-expressing iNeurons was

455 significantly increased in KO clones (Fig. 4G). When grouping iNeurons by genotype, KO

456 neurons were almost twice as likely to express miniature events (Fig. 4G). mEPSC frequency

457 was low and variable at this early time point, making it difficult to compare clones or even

458 genotypes (Fig. 4H-I). In contrast, $m$ EPSC amplitude was less variable. There appeared to be a 459 weak clonal and genotype effect on mEPSC amplitude. Both KO amplitude populations 460 exhibited a rightward shift compared to the two WT populations (Fig. 4J). When clonal data was 
461

462

463

464

465

466

467

468

469

470

471

472

473

474

475

476

477

478

479

480

481

482

483

484

485

486

487

488

489

490

491

492

493

494

collapsed by genotype, a robust statistical effect emerged at the level of individual events and at the level of cellular population means (Fig. 4J).

We next analyzed synaptic activity in more mature iNeurons (DIV40-50; Fig. 4L). As a population, neurons derived from WT clones were roughly twice as likely to express synaptic activity at this time point compared to younger neurons of the same genotype, indicative of substantial neuronal maturation during this period (Fig. 4G, M). However, this effect was less pronounced in KO neurons (Fig. 4G, M). There was a significant effect of time on the proportion of neurons expressing synaptic activity in WT neurons, but this effect was absent in KO iNeurons (Fig. 4M). There was no longer an effect of genotype on the proportion of neurons with synaptic activity at the more mature stage of development. Within the population of neurons with synaptic events, we measured mEPSC frequency and amplitude. The frequency of events was highly variable in these populations (Fig. $\mathbf{4 N - O}$ ), which made it difficult to draw clear conclusions across clones and genotypes. There was a trend toward more frequent events in combined KO populations, though these trends were not apparent when looking at individual clones. With respect to amplitude (Fig. 4P-Q), we once again observed a weak effect of clone and genotype at this timepoint that was consistent with observations from developmentally younger iNeurons. Neurons from both $\mathrm{KO}$ clones appeared to have slightly larger events compared to those from WT iNeurons. This effect was apparent in comparisons of mEPSC distributions of all events (Fig. 4P-Q), and in the much less sensitive approach of comparing event means from individual neurons (Fig. 4P-Q, inset).

The effect of SynGAP expression on iNeuron $m$ EPSC frequency and amplitude was somewhat consistent across developmental time points, but the effect sizes, when present, were relatively small. To determine if these effects were reproducible, we performed an additional experiment on iNeurons produced from the same clones. Data for this experiment was collected from a completely new hiPSC expansion and neuronal induction procedure. In this additional experiment, we observed similar effects of SynGAP expression on intrinsic membrane properties and mEPSCs (Fig. 5A-G). SYNGAP1 deletion did not affect the resting membrane potential, input resistance, or capacitance at the clonal or genotype level (Fig. 5A-C). Analysis of $\mathrm{mEPSC}$ frequency from each of the clones revealed a trend for increased frequency from neurons with disruptive SYNGAP1 variants (Fig. 5D). The two KO clones have a greater frequency of mEPSCs when looking at cumulative probability distributions and this drove an effect at the genotype level (Fig. 5E). A statistical effect was not present when comparing 
cellular means of mEPSC frequency. For mEPSC amplitude, the clonal and genotype effects were clearer compared to frequency measures. The cumulative distribution for mEPSC amplitudes for all events clearly shifted to larger values in both $\mathrm{KO}$ clones (Fig. 5F). This drove a substantial and highly significant shift in the disruption at the genotype level (Fig. 5G). We did not observe an effect on population means when looking at cellular averages. However, the power for this experiment was lower than the one presented in Figure 4. Taken together, we conclude that reducing SynGAP expression in Ngn2 iNeurons leads to weak, but reproducible, effects on mEPSC amplitude. Effects on frequency were unclear due to high variability.

Our data demonstrate that reducing SynGAP expression results in larger iNeurons that exhibit early synaptic maturity. Therefore, we hypothesized that reducing SynGAP expression would also influence the development of network activity in cultured iNeurons. To test this, we measured spontaneous distributed network activity in cultures derived from KO and WT clones using a multielectrode array (MEA) system (Fig. 6A-C). Recordings of the same cultures were performed over the course of several weeks, which enabled in vitro measurements of network spiking activity during neuronal development. From as early as week 2 , we observed evidence of spiking activity in cultures derived from each of the iPSC clones. However, both SYNGAP1 $\mathrm{KO}$ clones exhibited substantially increased firing rates compared to isogenic controls. The enhanced firing rate in $\mathrm{KO}$ iNeurons emerged progressively and was sustained through week six in culture at both clonal (Fig. 6D) and genotype levels (Fig. 6E). Next we measured bursting activity in each of the four clones. We observed significantly elevated neuronal bursts in KO vs control neurons (Fig. 6F-G). Quantification of distributed network connectivity demonstrated that KO neuronal cultures displayed different degrees of neural network activity, observed as "network bursts", as early as 3 weeks of maturation. Enhanced network bursting activity in KO cultures relative to WT controls was observed at both the clonal (Fig. 6H) and genotype levels (Fig. 6I). Thus, SYNGAP1 expression substantially influences the dynamics of cellular activity in developing neuronal networks. 


\section{Discussion}

525 We produced iNeurons from human hiPSCs with a disrupted SYNGAP1 gene in an effort to

526 understand how this gene shapes human neuron development and function. This is an

527 important research question given that pathogenic SYNGAP1 variants cause a complex

528 neurodevelopmental disorder defined by early-onset epilepsy, cognitive impairment, and autistic

529 features (Hamdan et al., 2011; Jimenez-Gomez et al., 2019; Vlaskamp et al., 2019; Satterstrom

530 et al., 2020). We found that SYNGAP1 regulates the postmitotic maturation of dendrites and

531 synapses from human iNeurons. Cas9-mediated disruption of SYNGAP1 expression enhanced

532 dendritic morphogenesis, accelerated the acquisition of synaptic activity, and drove increased

533 spiking activity measured in functionally connected two-dimensional iNeuron cultures. Our data

534 indicate that loss of SynGAP protein expression was responsible for the dendrite and synapse

535 maturation phenotypes observed in these cultures. Indeed, we observed consistent structural

536 phenotypes at the level of individual clones that were subsequently grouped by genotype.

537 Whole exome sequencing demonstrated that the only shared variants between the two KO

538 clones were indels in the SYNGAP1 gene, and immunoblotting confirmed that iNeurons derived

539 from KO clones expressed nominal levels of SynGAP protein. Altered dendritic maturation was

540 supported by data obtained from orthogonal experimental measures. We observed longer

541 dendrites in eGFP-positive iNeurons, and an increased dendritic area measured from

542 endogenous MAP2 signal. iNeurons derived from the KO hiPSC clone also exhibited an

543 increase in the absolute density of postsynaptic structures, a finding consistent with a neuronal

544 culture populated with neurons containing longer dendrites. Given that the length of dendrites

545 and the density of postsynaptic structures in iNeurons increases over time in culture (Zhang et

546 al., 2013), these data support the conclusion that SynGAP expression regulates the maturation

547 rate of dendritic and synaptic structures in human iNeurons. This conclusion was also supported

548 by clonal and genotype differences in synaptic activity between WT and KO iNeurons. Individual

549 iNeurons have been shown to gradually acquire synaptic activity in the first several weeks in

550 culture (Zhang et al., 2013; Nehme et al., 2018). However, we found that KO neurons

551 expressed synaptic activity earlier in development compared to WT neurons.

552

553 Distributed neuronal activity, measured by MEA analysis, confirmed that structural maturation of

554 dendrites and early functional expression of synapse activity translated into increased network

555 activity in KO cultures. Similar to what we observed in dendrites and synapses, measures of

556 network activity normally observed in more mature WT cultures appeared at much earlier stages

557 of development in neurons developed from KO clones. Activity was already substantially greater 
558 in cultures derived from $\mathrm{KO}$ clones at two weeks, a time in development when there is very little

559 activity present in WT cultures. In addition, statistical analysis of network activity that considered

560 time as a factor demonstrated that the trajectory of neuronal activity was distinct in KO cultures

561 compared to WT controls (Fig. 6). Indeed, activity increased at a much greater rate in KO

562 cultures, compared to WTs, over the first several weeks of development. Networks formed from

563 iNeurons exhibited bursting behavior as a function of time in vitro, with older cultures exhibiting

564 more robust bursting behavior (Fischer). Network bursting is driven in part by increased

565 functional synaptic connectivity among neurons (Suresh et al., 2016; Nehme et al., 2018). KO

566 neurons extended dendrites more quickly and had greater numbers of postsynaptic structures.

567 Thus, KO neurons would be expected to exhibit enhanced connectivity at younger ages

568 compared to control cultures. Increased functional connectivity in KO networks, driven by longer

569 dendrites with more synaptic structures likely contributed to the precocious onset of coordinated

570 network bursting behavior observed in MEA experiments. The effects observed on network

571 activity were apparent at the level of individual clones when grouped by SYNGAP1 genotype.

572 These data further strengthen the conclusion that loss of SynGAP protein drives effects on

573 network activity and these data provide a possible neurobiological mechanism for why

574 individuals with SYNGAP1 mutations have such a high incidence of early onset pediatric

575 seizures (Vlaskamp et al., 2019).

576

577 Data implicating SYNGAP1 expression on the structural and functional maturation of human

578 neurons is consistent with known functions of this gene discovered from experimentation in

579 mouse neurons (Kilinc et al., 2018). SynGAP protein is highly expressed in rodent neurons and

580 is capable of bidirectional regulation of excitatory synapse strength. Overexpression of SynGAP

581 protein suppresses excitatory synapse transmission by activating AMPA receptor internalization

582 (Rumbaugh et al., 2006). One report indicates that SynGAP isoforms regulate synaptic strength

583 in opposing directions (McMahon et al., 2012). However, genetic ablation of all Syngap1 splice

584 forms in mice, which removes expression of all protein isoforms, leads to increased excitatory

585 synapse strength and early appearance of synaptic activity in glutamatergic neurons (Clement

586 et al., 2012). These data indicate that the integrated function of all SynGAP proteins in

587 developing mouse neurons is to suppress excitatory synapse function during development. Our

588 findings in human neurons, which also ablated expression of human SynGAP isoforms, support

589 this model of developmental SYNGAP1 function. Given that we observed early and enhanced

590 excitatory synapse function in KO iNeurons, human SYNGAP1 also appears to slow the onset

591 of excitatory synapse activity by suppressing excitatory synapse function. 
593 The impact of SYNGAP1 on human neuron dendritic maturation is also consistent with

594 observations in rodent neurons. The effect of SynGAP protein expression on rodent neuron

595 dendritic development is complex and depends on the type of neuron and brain area studied.

596 Syngap1 heterozygous KO mice have well documented impairments in dendritic morphogenesis

597 that is linked to alterations in neural circuit assembly and neuronal connectivity. Layer 5 (L5)

598 neurons in the somatosensory cortex of these mutant mice undergo a form of accelerated post-

599 mitotic differentiation, where dendritic extension proceeds at a quicker pace compared to WT

600 mice (Aceti et al., 2015). Interestingly, these neurons also undergo premature spine

601 morphogenesis and early spine pruning. These observations, combined with a

602 desynchronization of L5 cell body and dendritic arbor growth, strongly indicate that SynGAP

603 expression acts in these neurons to suppress a differentiation program that stimulates neuronal

604 maturation. In contrast to these findings, neurons in the upper lamina (Layers 2-4) of the

605 somatosensory cortex of Syngap1 KO mice show the opposite phenotype. These neurons

606 undergo a form of arrested development where dendritic arbors are shorter compared to similar

607 neurons in WT littermates (Michaelson et al., 2018). Neurons with shorter dendritic arbors also

608 had fewer dendritic spines and these structural alterations impacted connectivity within

609 somatosensory cortex circuits. While our studies in human iNeurons support a role for

610 SYNGAP1 to suppress dendritic maturation, the specific effect of the gene on structural

611 maturation may also be dependent on the type of human neuron. Two-dimensional neuronal

612 cultures lack the cellular complexity of neural networks found in the intact nervous system. It will

613 be of considerable interest to assess how loss of SYNGAP1 expression impacts various types

614 of genetically and morphologically distinct neurons formed in three-dimensional human culture

615 systems, such as organoids, and how alterations to dendritic morphogenesis may contribute to

616 impaired neural circuit connectivity and development of network activity.

618 Acknowledgments

619 Funding: This work was supported in part by NIH grants from the National Institute of Mental

620 Health (MH096847 and MH108408 to G.R.) and the National Institute for Neurological Disorders

621 and Stroke (NS064079 and NS110307 to G.R. and NS091381 to J.H.). N.L. was supported by a

622 generous postdoctoral training fellowship and J.H. by a grant from the SynGAP Research Fund

623 (SRF; https://syngapresearchfund.org). This work was supported by Grant 201763 from the

624 Doris Duke Charitable Foundation to J.H. V.A. was supported by a generous postdoctoral 
625 training fellowship from Leon and Friends e.V (https://leonandfriends.org). L.B. was partially

626 supported by a travel grant for junior researchers from Boehringer Ingelheim Fonds (BIF). J.H.

627 also received generous support from the Robbins Foundation and Mr. Charif Souki.

628 Author Contributions: N.L. performed experiments, designed experiments, analyzed data, co-

629 wrote and edited the manuscript. V.A, R. V., M.K., L.B., C.R., A.R., C.H., B.S., and E.W.

630 performed experiments, analyzed data and interpreted data. G.R. conceived project, designed

631 experiments, interpreted data, and co-wrote the manuscript. D.R.P., L.S., T.P.S., C.A.M., and

632 J.L.H. designed and interpreted experiments and edited the manuscript.

633 Competing interests: E.W. and D.R.P. are employed by Thermo Fisher Scientific. The authors

634 declare no competing interests.

635 Data and materials availability: hiPSC clones and data supporting the findings of this study

636 are available from the corresponding author upon reasonable request. 
bioRxiv preprint doi: https://doi.org/10.1101/2020.06.01.127613; this version posted June 3, 2020. The copyright holder for this preprint (which was not certified by peer review) is the author/funder, who has granted bioRxiv a license to display the preprint in perpetuity. It is made available under ACC-BY-NC-ND 4.0 International license.

Figures and Legends

\section{Figure 1}

A

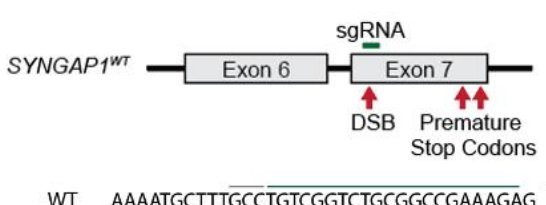

WT AAAATGCTTTGCCTGTCGGTCTGCGGCCGAAAGAG
B

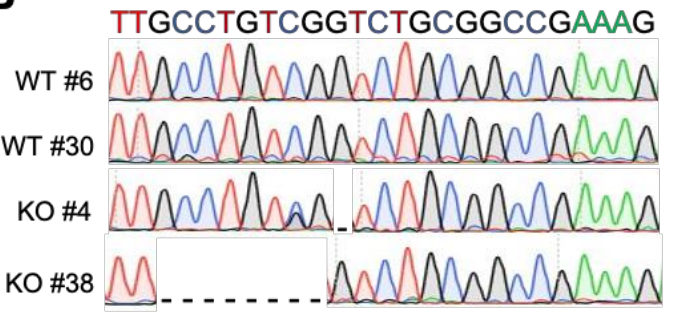

C

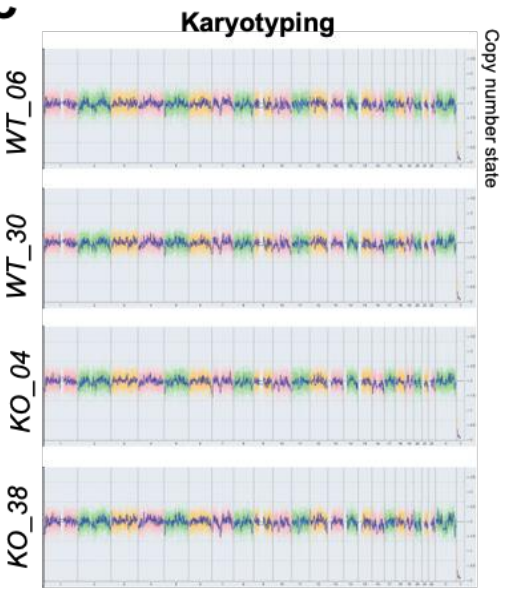

F $\quad \underline{\text { Total }} \stackrel{\underline{\text { Indel }}}{\underline{\text { count }}}$

Sample $\underline{\text { count }} \underline{\underline{\text { above }}}$

WT_06 70

WT_30 $10 \quad 0$

KO_04 $14 \quad 1$

KO_38 $13 \quad 1$

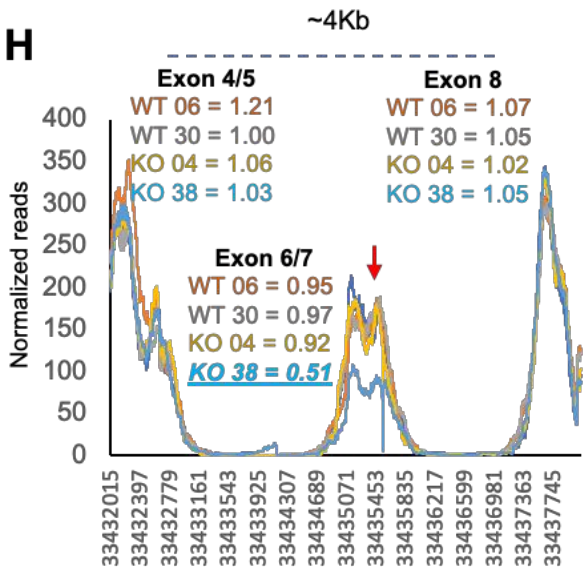

\section{Location} (chrom:loc)

NA

NA

$6: 33435551$

$6: 33435544$
D
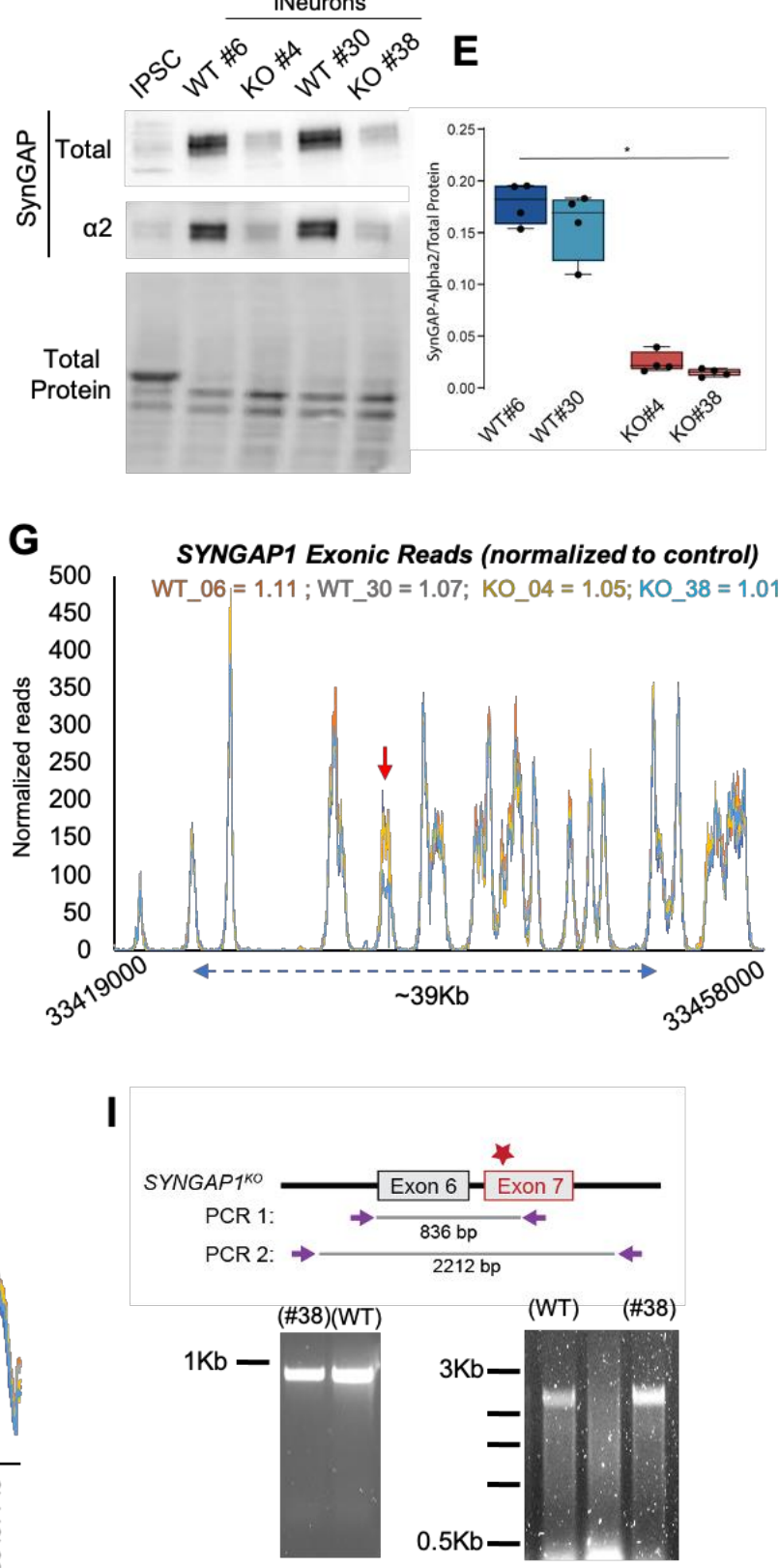
Figure 1. Development of isogenic SYNGAP1 knockout hiPSCs.

640 (A) Cartoon showing clone-specific mutations in the SYNGAP1 gene. (B) Sanger sequencing 641 for one WT clone and two SYNGAP1 mutant clones derived from the CRISPR experiment. (C)

642 Whole genome view of iNeurons from WT\#6, WT\#30, KO\#4 and KO\#38 clones depicting a

643 copy number value of 2 cross all chromosomes (expect for the $Y$-chromosome which is not

644 detected) revealing normal (female) karyotype with no chromosomal aberrations. The pink,

645 green and yellow colors indicate the raw signal for each individual chromosome probe, while the

646 blue signal represents the normalized probe signal which is used to identify copy number and

647 aberrations (if any). (D) Western blots demonstrating SynGAP protein expression from iNeuron

648 or iPSC homogenate. Total refers to signal from an antibody that detects all splice variants and

$649 \alpha 2$ refers to signal from an antibody that detects only a specific C-terminal splice variant. (E)

650 Quantification of relative intensity of bands normalized to total protein signal. One-way ANOVA

651 with a Kruskal-Wallis test multiple comparisons test $\mathrm{H}(3)=12.29, \mathrm{p}=0.0001$; WT\#6 vs KO\#4:

$652 p=0.1876$; WT\#6 vs WT\#30: $p>0.9999$; WT\#6 vs KO\#38: $p=0.0140$; KO\#4 vs WT\#30: $p=0.5258$;

653 KO\#4 vs KO\#38: p>0.9999; WT\#30 vs KO\#38: $p=0.0561 . n=4$ per group. In box-and-whisker

654 plot, the center, boxes and whiskers represent the median, interquartile range, and min to max,

655 respectively. (F) Indels from each clone identified from whole exome sequencing analysis.

656 Indels were identified by clonal sequence differences from the original Cas 9 hiPSCs (reference

657 sequence). Indel threshold was determined by at least $50 \%$ of the reads differing from the

658 reference sequence with a minimum of at least ten reads. Indels $w /$ frequency above 0.8 were

659 used to determine frequency of homozygous varaints. (G) Normalized mapped reads from the

660 entire coding sequence of the SYNGAP1 gene in the four clones hiPSCs. Red arrow denotes

661 predicted Cas9 cut site. Numbers reflect clonal reads relative to Cas9 hiPSC reads. (H)

662 Normalized mapped reads for the same samples around the Cas 9 target sequence. (I) Genomic

663 PCR to amplify DNA sequence flanking the Cas9 target site. 


\section{Figure 2}

A
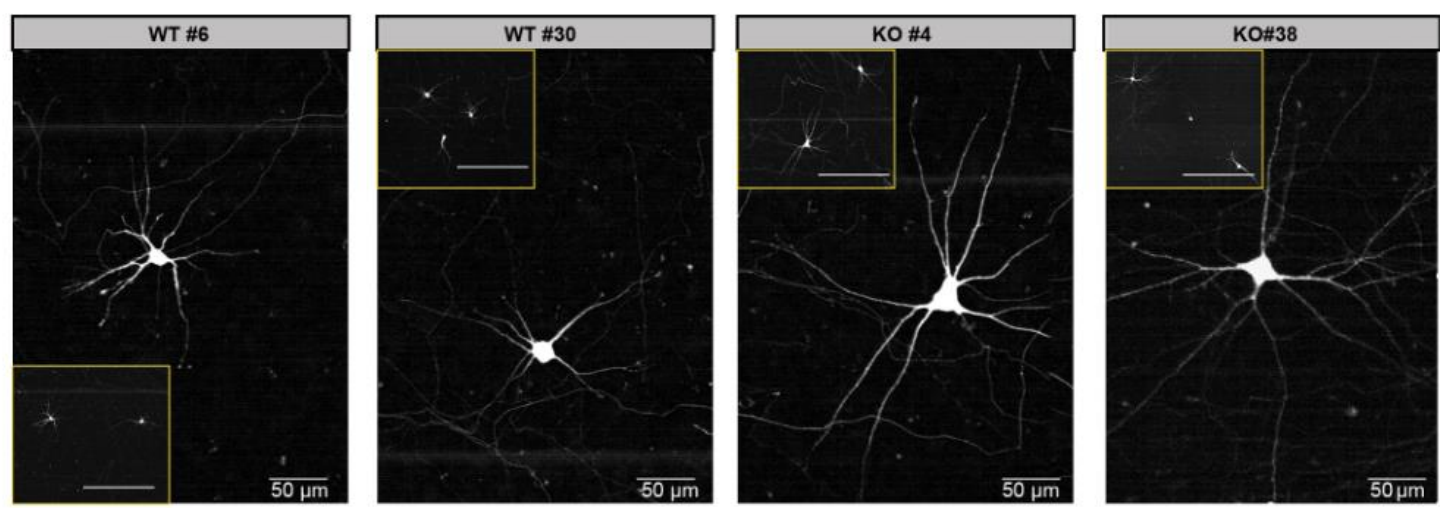

B

Total

Primary
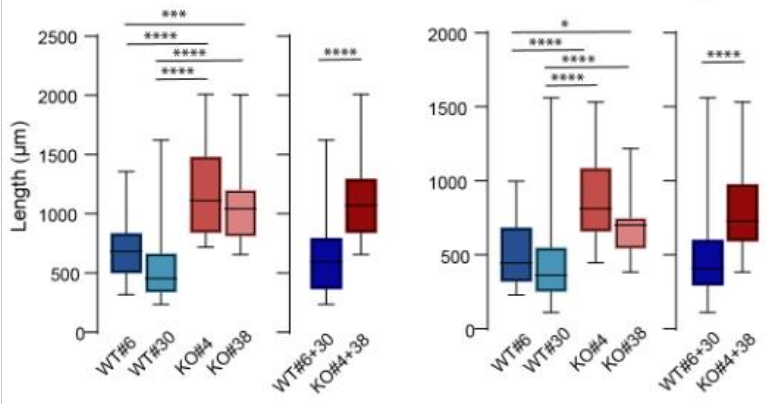

Secondary

Tertiary

C

Total

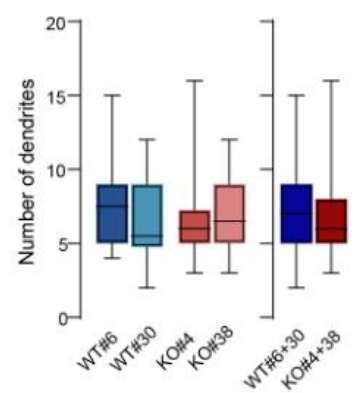

Primary
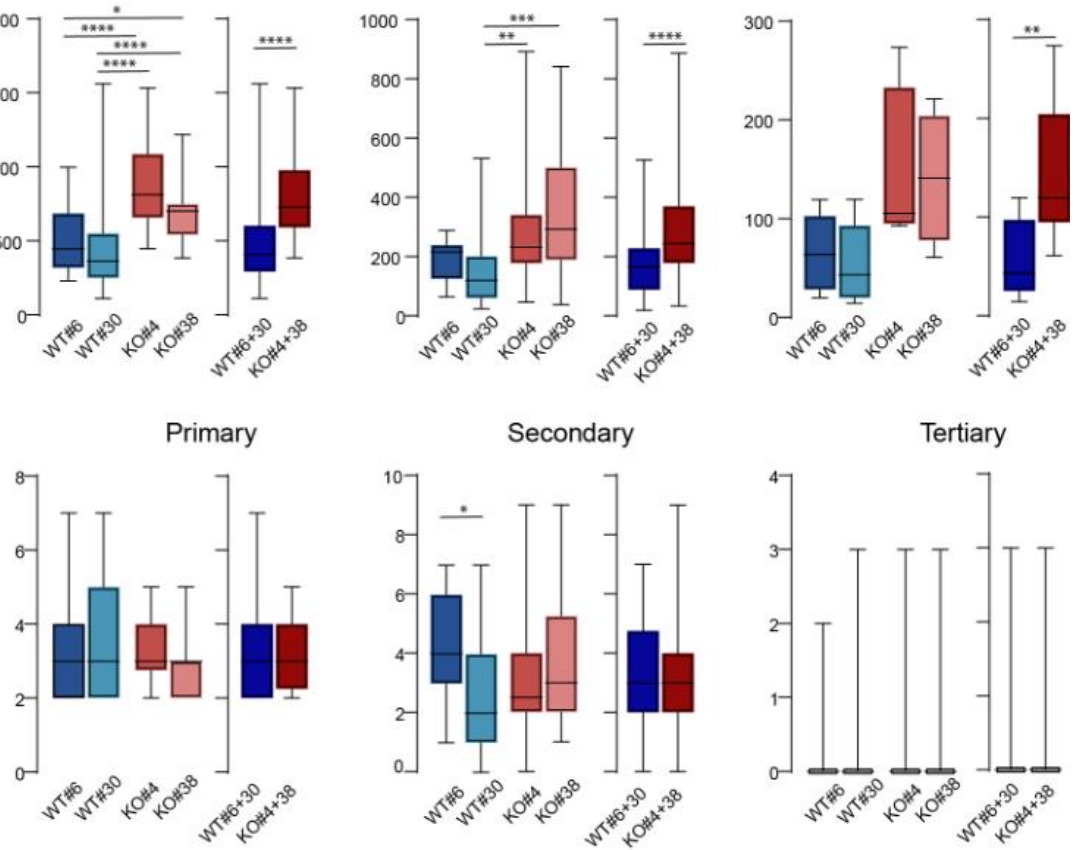

Secondary
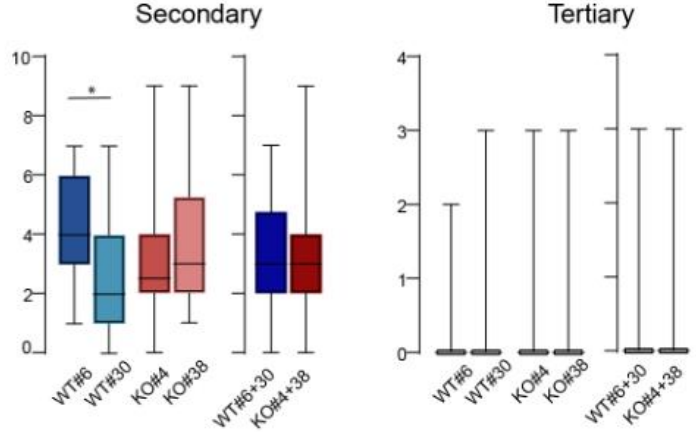

Figure 2. Increased dendrite length in iNeurons derived from KO iPSC clones. (A)

667 Representative images of eGFP-expressing iNeurons from the four different clones at DIV45

668 (inset images scale bars: $200 \mu \mathrm{m}$ ). (B-E) Histograms depicting average length per cell of total

669 (B), primary (C), secondary (D) and tertiary dendrites (E) of the four clones (Total dendrites -

670 clonal analysis $\mathrm{H}=54.81, \mathrm{p}<0.0001 ; \mathrm{N}=30$ cells per clone; Genotype analysis $\mathrm{U}=436, \mathrm{p}<0.0001$;

$671 \mathrm{~N}=60$ cells per genotype; Primary dendrites - Clonal analysis $\mathrm{H}=49.71, \mathrm{p}<0.0001, \mathrm{~N}=30$ cells

672 per clone; Genotype analysis $U=545, p<0.0001, N=60$ cells per genotype; Secondary dendrites

673 - Clonal analysis $\mathrm{H}=20.45, \mathrm{p}<0.0001 ; \mathrm{N}=30$ cells for WT\#6 and KO\#38; $\mathrm{N}=26$ cells for WT\#30;

$674 \mathrm{~N}=27$ cells from KO\#4; Genotype analysis $U=880, p<0.0001 ; N=56$ cells from WT genotype; 
$675 \mathrm{~N}=57$ cells from KO genotype; Tertiary dendrites - Clonal Analysis, $H=7.115, p=0.0683 ; N=6$

676 dendrites of 30 cells from WT\#6, $n=5$ dendrites of 30 cells from WT\#30, n=4 dendrites of 30

677 cells from KO\#4 and $n=4$ dendrites of 30 cells from KO\#38; Genotype analysis, $U=73.55$,

$678 \mathrm{p}=0.0068 ; \mathrm{N}=11$ cells from WT genotype; $\mathrm{N}=8$ cells from KO genotype). (F-I) Graphs showing

679 average number of dendrites per cell of total $(\mathbf{F})$, primary $(\mathbf{G})$, secondary $(\mathbf{H})$ and tertiary (I)

680 dendrites of the four clones (Total dendrites - Clonal analysis, $\mathrm{H}=5.957, \mathrm{p}=0.1137 ; \mathrm{N}=30$ cells

681 per clone; Genotype analysis, $\mathrm{U}=1613, \mathrm{p}=0.3222 ; \mathrm{N}=60$ cells per genotype; Primary dendrites -

$682 \mathrm{H}=1.680, p=0.6413, n=30$ cells per clone; Genotype analysis, $U=1639, p=0.3755, n=60$ cells per

683 genotype; Secondary dendrites - Clonal analysis, $H=10.72, p=0.0133, n=30$ cells per clone for

684 clone comparisons; Genotype analysis, $U=1689, p=0.5552, n=60$ cells per genotype; Tertiary

685 dendrites - Clonal analysis, $\mathrm{H}=0.4531, \mathrm{p}=0.9291, \mathrm{n}=30$; Genotype analysis, $\mathrm{U}=1731, \mathrm{p}=0.6129$,

$686 \mathrm{n}=60$ cells per genotype). In box-and-whisker plots, the center, boxes and whiskers represent

687 the median, interquartile range, and $\min$ to $\max$, respectively. ${ }^{*} p<0.05,{ }^{* *} p<0.01,{ }^{* * *} p<0.0001$. 


\section{Figure 3}
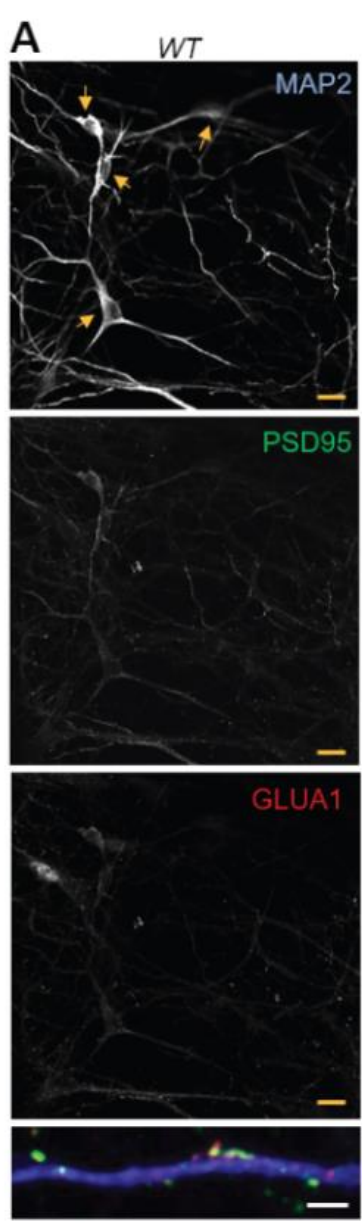
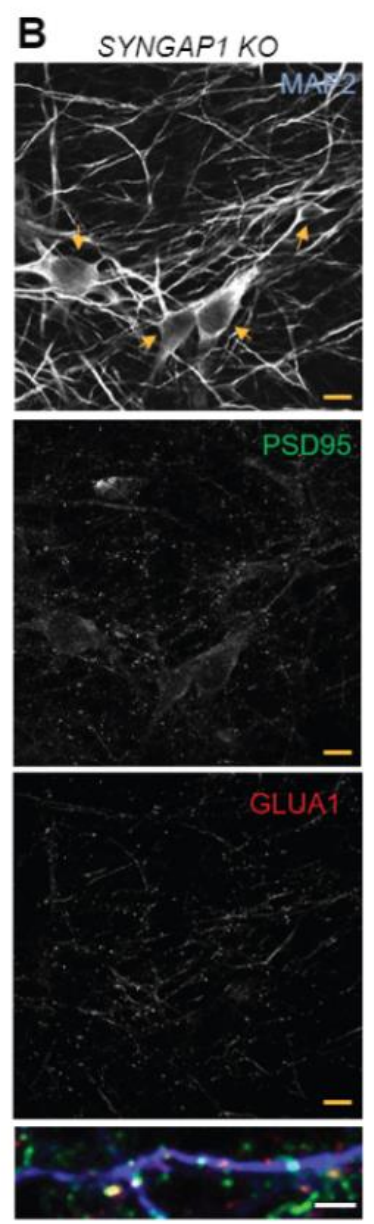

689

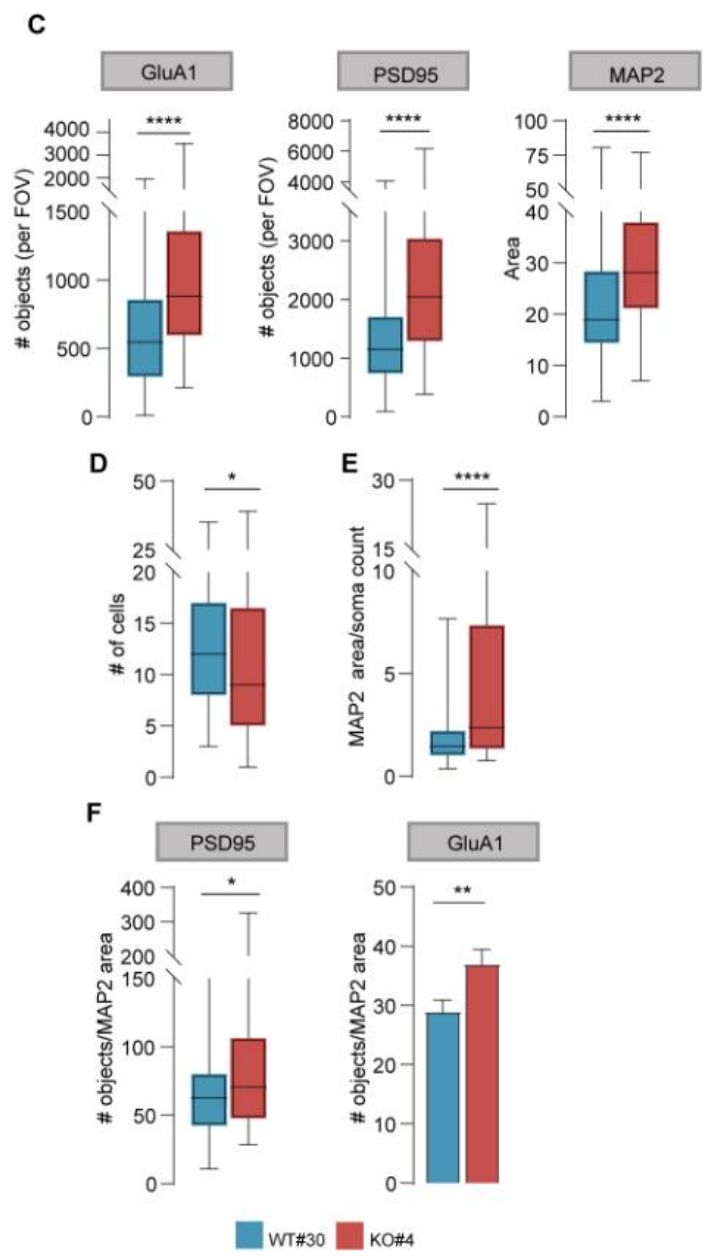

Figure 3. Increased dendritic area and more numerous postsynaptic structures in

SYNGAP1 KO iNeurons. (A-B) Representative images showing MAP2 labeling (top), PSD95 labeling (middle), and GluA1 labeling (middle-bottom), and merge of MAP2, PSD95 and GLUA1 (bottom) of iNeurons from WT\#30 (A) and KO\#4 (B) at DIV45. Yellow arrows indicate cell bodies. Yellow scale bar is $10 \mu \mathrm{m}$ and white is $2 \mu \mathrm{m}$. (C) Graphs showing MAP2 area and number of PSD95 objects (punctate labeling) per field of view (FOV) in WT\#30 and KO\#4. (MAP2 comparison: $U=2181, p<0.0001 \mathrm{~N}=85$ images for WT\#30 and 81 images for KO\#4; PSD95 comparison: $U=1891, p<0.0001 \mathrm{~N}=85$ for WT\#30 and 81 for KO\#4; GluA1 comparison: $\mathrm{U}=1999, \mathrm{p}<0.0001 \mathrm{~N}=85$ for WT\#30 and 81 for KO\#4) (D) Averaged number of cells in FOVs WT\#30 and KO\#4 (U=2746, p=0.0240 N=85 for WT\#30 and 81 for KO\#4). (E) MAP2 area by soma count $(U=2097, p<0.0001 \mathrm{~N}=85$ for WT\#30 and 81 for KO\#4). (F) Graphs showing quantification of PSD95 and GluA1 expression in WT\#30 and KO\#4 normalized to MAP2 area (PSD95 objects/MAP2 area comparison: $U=2752, p=0.0255 \mathrm{~N}=85$ for WT\#30 and 81 for KO\#4; 
bioRxiv preprint doi: https://doi.org/10.1101/2020.06.01.127613; this version posted June 3, 2020. The copyright holder for this preprint (which was not certified by peer review) is the author/funder, who has granted bioRxiv a license to display the preprint in perpetuity. It is made available under aCC-BY-NC-ND 4.0 International license.

703 GluA1 objects/MAP2 area comparison: $t_{(164)}=2666, p=0.0084 \mathrm{~N}=85$ for WT\#30 and 81 for

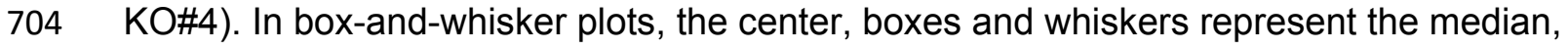

705 interquartile range, and min to max, respectively. Bar graph represents mean $\pm \mathrm{SEM}$. ${ }^{*} p<0.05$,

$706{ }^{* *} p<0.01$ and ${ }^{* * * *} p<0.0001$

707

708 
bioRxiv preprint doi: https://doi.org/10.1101/2020.06.01.127613; this version posted June 3, 2020. The copyright holder for this preprint (which was not certified by peer review) is the author/funder, who has granted bioRxiv a license to display the preprint in perpetuity. It is made available under ACC-BY-NC-ND 4.0 International license.

Figure 4

A

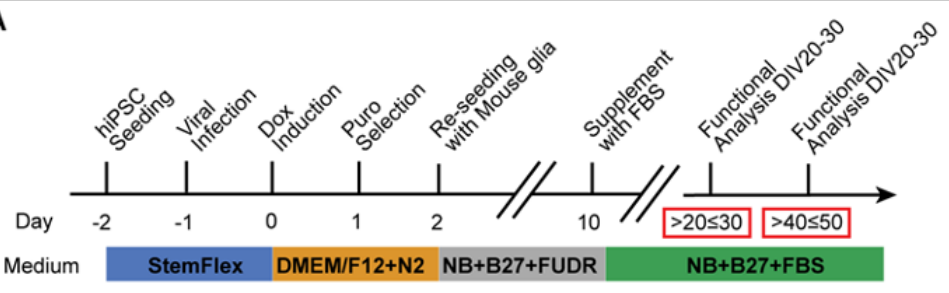

B

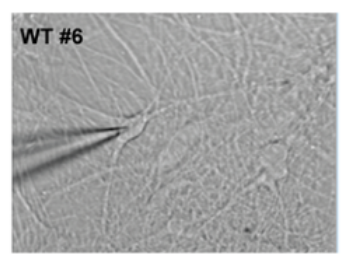

I
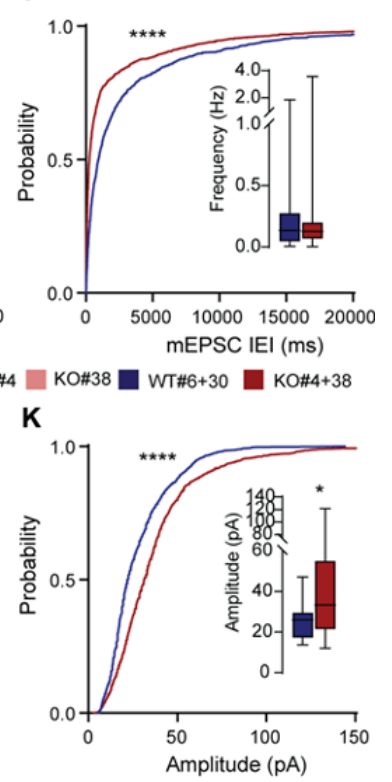

。

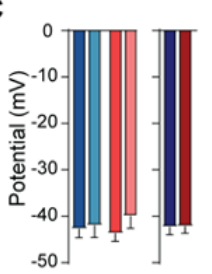

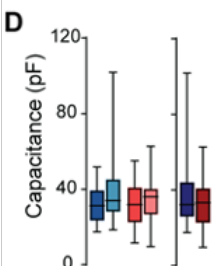

E

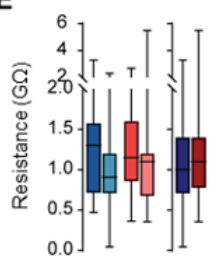

F

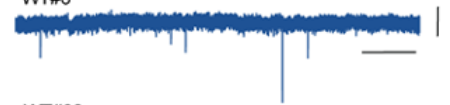

WT\#30

KO\#4

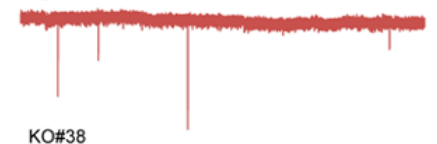

KO\#38
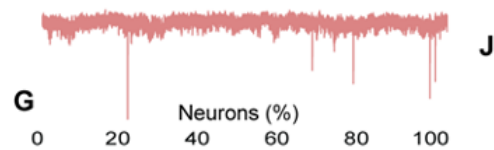

$\mathrm{H}$

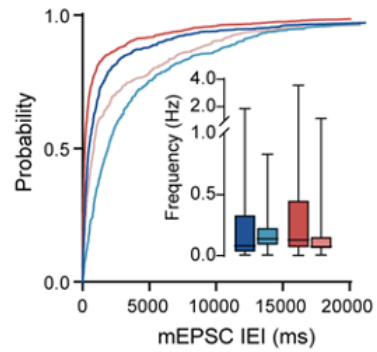

J

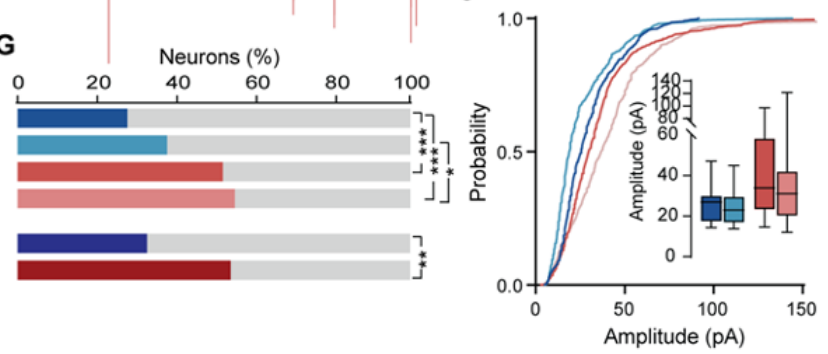

DIV40-50

L $\quad$ WTH6

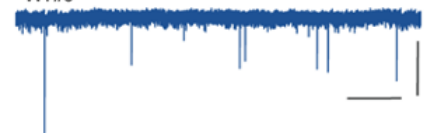

WT $\# 30$

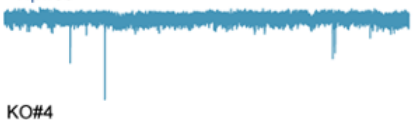

KO\#4

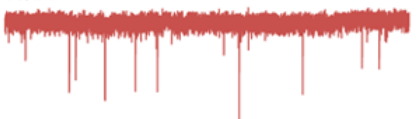

KO\#38

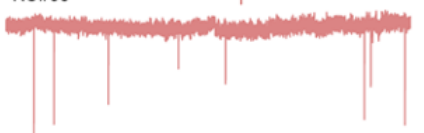

M

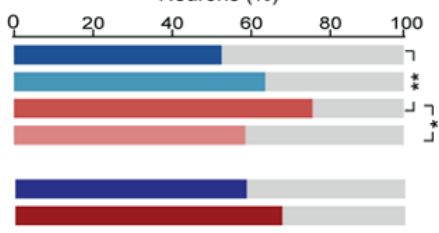

N

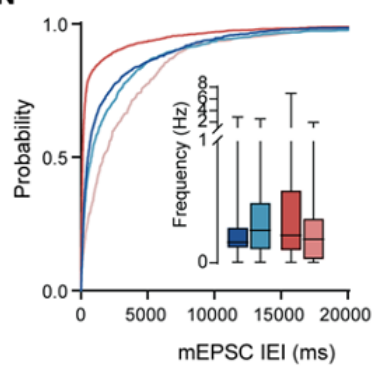

O

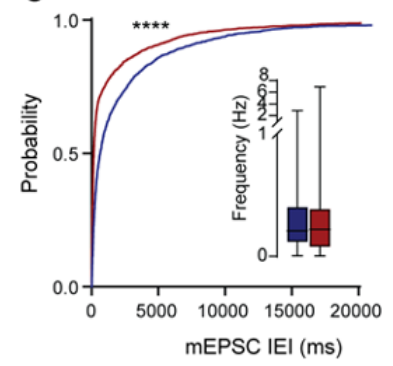

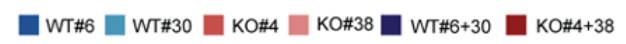

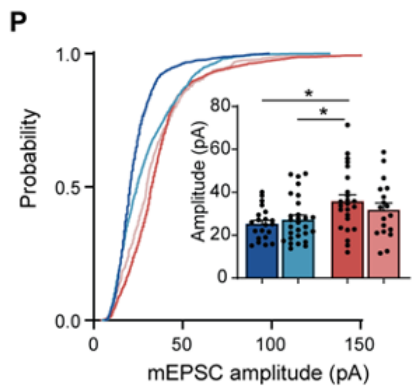

Q

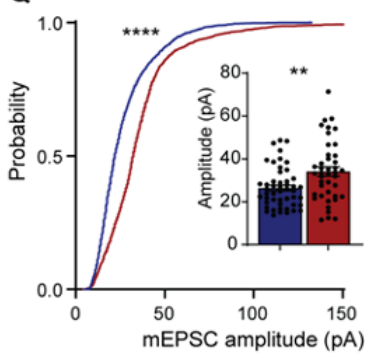


710

711

712

713

714

715

716

717

718

719

720

721

722

723

724

725

726

727

728

729

730

731

732

733

734

735

736

737

738

739

740

741

742

743

\section{Figure 4. SYNGAP1 expression in human iNeurons regulates excitatory synapse}

function. (A) Flow diagram of iNeuron generation from WT and SYNGAP1 KO iPSCs for whole-cell electrophysiological experiments (recording days within red boxes). (B) Representative DIC image of patched iNeurons cells from WT\#6. (C-E) Bar graphs representing intrinsic membrane properties measured at DIV20-30 as resting membrane potential (C), capacitance (D) and input resistance (E) from the four clones (Membrane potential - Clonal analysis, $F_{(3.95)}=0.5132, p=0.6742, n=29$ cells from WT\#6, 31 cells from WT\#30, 34 cells from $\mathrm{KO \# 4}$ and 21 cells from KO\#38;Genotype analysis, $\mathrm{t}_{(97)}=0.08684, \mathrm{p}=0.9310, \mathrm{n}=51$ cells for WT\#6+30 and 48 for KO\#4+38; Capacitance - Clonal analysis, $H=3.123, p=0.3730, n=29$ cells from WT\#6, 31 cells from WT\#30, 34 cells from KO\#4 and 21 cells from KO\#38; Genotype analysis, $U=1584, p=0.5093 ; N=62$ cells from $W T \# 6+30$ and 55 cells from $\mathrm{KO} \#+38$;

Membrane resistance- Clonal analysis, $\mathrm{H}=4.259, \mathrm{p}=0.2348, \mathrm{n}=28$ cells from WT\#6, 31 cells from WT\#30, 34 cells from KO\#4 and 21 cells from KO\#38; Genotype analysis, U=1546, $p=0.5619 ; N=60$ cells from WT\#6+30 and 55 cells from KO\#4+38). (F) Representative traces of mEPSCs of iNeurons from WT and KO clones at DIV20-30. Scale bars 2 s, 20pA. (G)

Percentage of successful observations of mEPSCs in iNeurons from the four clones at DIV2030 (Clonal analysis, $p=0.0008$ for KO\#4 vs WT\#6; $p=0.0002$ for KO\#38 vs WT\#6, $p=0.0644$ for KO\#4 vs WT\#30, $n=20$ for KO\#4 and 18 for WT\#30, resp; $p=0.0231$ for KO\#38 vs WT\#30; $N=$ 12 cells from WT\#6, 18 cells from WT\#30, 20 cells from KO\#4 and 19 cells from KO\#38;

Genotype analysis, $p=0.0042$ for $K O \# 4+38$ vs WT\#6+30, $n=30$ cells from WT\#6+30 and 39 cells from KO\#4+38). ( $\mathrm{H}-\mathrm{I})$ Cumulative plots of mEPSC interevent-interval and frequency (inset) of the different clones individually $(\mathbf{H})$ and grouped by genotype $(\mathbf{I})$ at DIV20-30 (Clonal analysis, $\mathrm{H}=1.910, p=0.5912, \mathrm{n}=12$ cells from WT\#6, 18 cells from WT\#30, 20 cells from KO\#4 and 19 cells from KO\#38; Genotype analysis, $U=504.5, p=0.5607 ; N=29$ cells from WT\#6+30 and 38 cells from KO\#4+38, K-S test $D=0.2660, p<0.0001, n=951$ events from 29 cells from WT\#6+30, $\mathrm{n}=1559$ events from 38 cells from KO\#4+38). (J-K) Cumulative probability plots of mEPSC amplitude of the different clones individually $(\mathbf{J})$ and grouped by genotype $(\mathbf{K})$ at DIV20-30 (Clonal analysis, $H=7.565, p=0.0559, n=15$ cells from WT\#6, 20 cells from WT\#30, 19 cells from KO\#4 and 19 cells from KO\#38; Genotype analysis, $U=504.5, p=0.5607 ; N=29$ cells from WT\#6+30 and 38 cells from KO\#4+38, K-S test $D=0.2660, p<0.0001, n=981$ events from 35 cells from WT\#6+30, $n=1601$ events from 38 cells from KO\#4+38). (L) Representative traces of mEPSCs of iNeurons from WT and KO clones at DIV40-50. Scale bars $2 \mathrm{~s}, 20 \mathrm{pA}$. (M)

Percentage of successful observations of mEPSCs in iNeurons from the four clones at DIV4050 (Clonal analysis, $p=0.0011$ for WT\#6 vs KO\#4; $p=0.4764$ for WT\#6 vs KO\#38; $p=0.0892$ for 
744 WT\#30 vs KO\#4; $p=0.5612$ for WT\#30 vs KO\#38; $p=0.1511$ for WT\#6 vs WT\#30; $p=0.0154$ for

$745 \mathrm{KO \# 4}$ vs KO\#38; $\mathrm{N}=21$ cells from WT\#6, 29 cells from WT\#30, 25 cells from KO\#4 and 18 cells

746 from KO\#38; Genotype analysis, $p=0.2399$ for $W T \# 6+30$ vs KO\#4+38; $N=50$ cells from

747 WT\#6+30 and 43 cells from KO\#4+38; Effect of time, $p=0.0004$ for WT\#6+30 p40 vs p20; $p=$

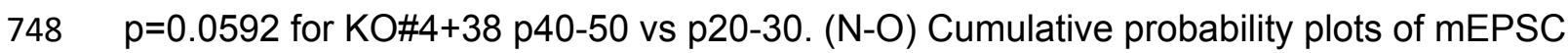

749 interevent-interval (IEI) and frequency (inset) of the different clones individually ( $N$ ) and grouped

750 by genotype $(\mathbf{O})$ at DIV40-50 (Clonal analysis, $\mathrm{H}=2.874, \mathrm{p}=0.4115, \mathrm{n}=21$ cells from WT\#6, 28

751 cells from WT\#30, 24 cells from KO\#4 and 18 cells from KO\#38; Genotype analysis, U=970.5,

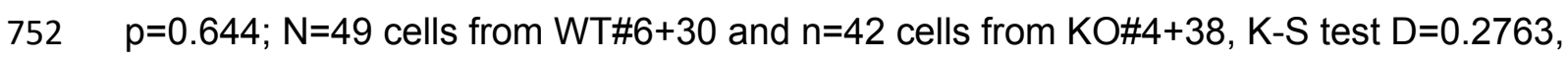

$753 \mathrm{p}<0.0001, \mathrm{n}=2182$ events from 49 cells from WT\#6+30, $n=2498$ events from 42 cells from

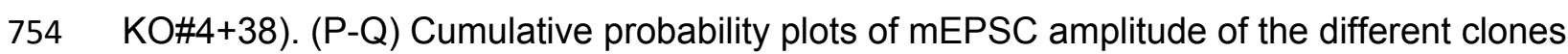

755 individually $(\mathbf{P})$ and grouped by genotype $(\mathbf{Q})$ at DIV40-50 (Clonal analysis, $F_{(3.87)}=3.73$,

$756 p=0.0142, p=0.0187$ for KO\#4 vs WT\#6, $p=0.0499$ for KO\#4 vs WT\#30, $p=0.9407$ for WT\#6 vs

757 WT\#30; $p=0.3151$ for WT\#6 vs KO\#38; $p=0.5696$ for WT\#30 vs KO\#4 and $p=0.70$ for KO\#4 vs

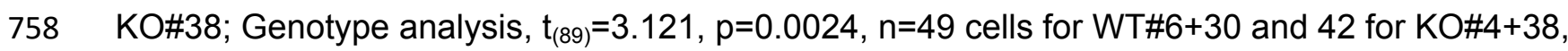

759 K-S test $D=0.2990, p<0.0001, n=2254$ events from 49 cells from $W T \# 6+30, n=2554$ events

760 from 42 cells from KO\#4+38). In box-and-whisker plots, the center, boxes and whiskers

761 represent the median, interquartile range, and $\min$ to max, respectively. Bar graphs represent

762 mean \pm SEM. ${ }^{*} p<0.05,{ }^{* *} p<0.01$ 


\section{Figure 5}

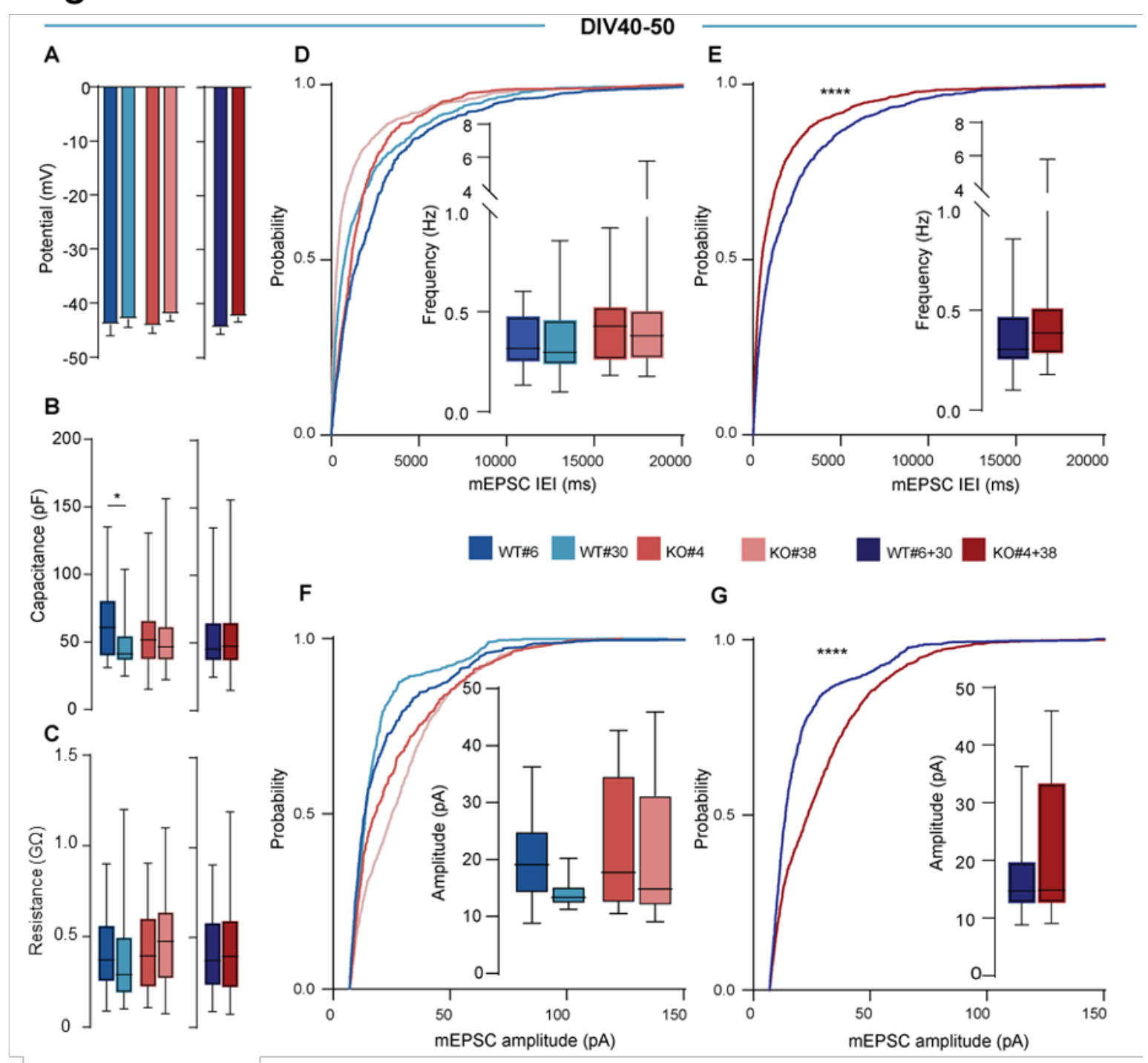

Figure 5. Reproducibility of SYNGAP1-mediated effects on iNeuron excitatory synapse

766

767

768

769

770

771

772

773

774

775

function. (A-C) Graphs showing resting membrane potential (A), capacitance (B) and input resistance (C) from the four clones at DIV40-50 (Membrane potential - Clonal analysis, $F_{(3.54)}=0.5456, p=0.6532, n=11$ cells from WT\#6, 16 cells from WT\#30, 13 cells from KO\#4 and 18 cells from KO\#38; Genotype analysis, $t_{(56)}=1.215, p=0.2295, n=27$ cells for WT\#6+30 and 21 for KO\#4+38; Capacitance - Clonal analysis, $H=9.091, p=0.0281, p=0.0318$ for WT\#6 vs WT\#30, $n=28$ cells from WT\#6, 41 cells from WT\#30, 34 cells from KO\#4 and 50 cells from KO\#38; Genotype analysis, $U=2828, p=0.7973 ; N=69$ cells from WT\#6+30 and 84 cells from KO\#4+38; Membrane resistance-Clonal analysis, $\mathrm{H}=4.738, \mathrm{p}=0.1920, \mathrm{n}=28$ cells from WT\#6, 41 cells from WT\#30, 34 cells from KO\#4 and 50 cells from KO\#38; Genotype analysis, $\mathrm{U}=2896, \mathrm{p}=0.9949 ; \mathrm{N}=69$ cells from WT\#6+30 and 84 cells from KO\#4+38 ).(D-E) Cumulative 
776 plots of mEPSC interevent-interval (IEI) and frequency (inset) of the different clones individually

777 (D) and grouped by genotype (E) at DIV40-50 (Clonal analysis, $\mathrm{H}=1.663, \mathrm{p}=0.6452, \mathrm{n}=11$ cells

778 from WT\#6, 9 cells from WT\#30, 8 cells from KO\#4 and 13 cells from KO\#38; Genotype

779 analysis, $\mathrm{U}=164, \mathrm{p}=0.2382 ; \mathrm{N}=20$ cells from $\mathrm{WT} \# 6+30$ and $\mathrm{n}=21$ cells from $\mathrm{KO} \# 4+38, \mathrm{~K}-\mathrm{S}$ test

$780 D=0.1744, p<0.0001, n=1199$ events from 20 cells from WT\#6+30, $n=1512$ events from 21

781 cells from KO\#4+38). (F-G) Cumulative probability plots of mEPSC amplitude of the different

782 clones individually $(\mathbf{F})$ and grouped by genotype $(\mathbf{G})$ at DIV40-50 (Clonal analysis, $\mathrm{H}=3.080$,

$783 \mathrm{p}=0.3795, \mathrm{n}=11$ cells from WT\#6, 9 cells from WT\#30, 8 cells from KO\#4 and 13 cells from

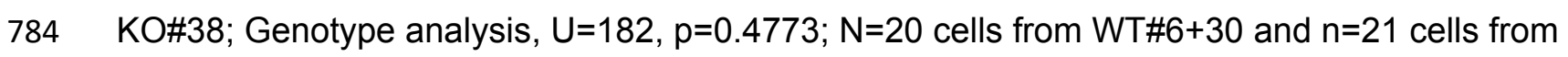

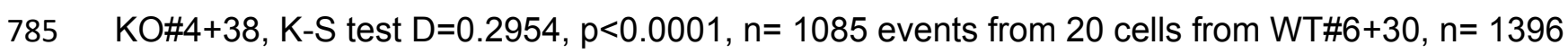

786 events from 21 cells from KO\#4+38). In box-and-whisker plots, the center, boxes and whiskers

787 represent the median, interquartile range, and $\min$ to max, respectively. Bar graphs represent

788 mean \pm SEM. * $p<0.05$. 
bioRxiv preprint doi: https://doi.org/10.1101/2020.06.01.127613; this version posted June 3, 2020. The copyright holder for this preprint (which was not certified by peer review) is the author/funder, who has granted bioRxiv a license to display the preprint in perpetuity. It is made available under aCC-BY-NC-ND 4.0 International license.

\section{Figure 6}
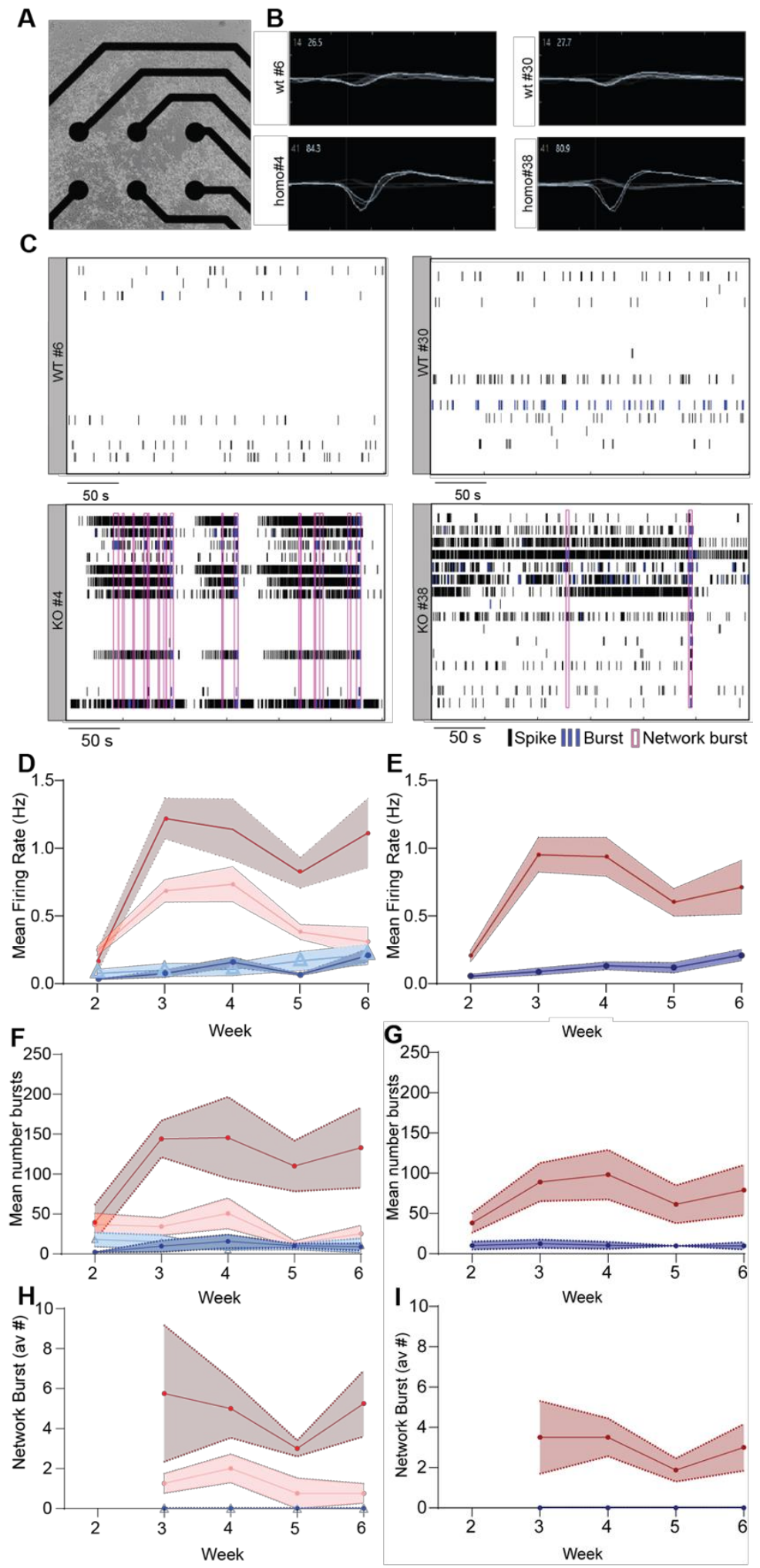

$\rightarrow$ WT\#6 $\rightarrow$ WT\#30 $\rightarrow \mathrm{KO \# 4}-\mathrm{KO \# 38}$

$\rightarrow \mathrm{WT} \# 6+30 \longrightarrow \mathrm{KO \# 4+38}$ 
Figure 6. Earlier onset and elevated levels of network activity in SYNGAP1 KO iNeurons.

792

793

794

795

796

797

798

799

800

801

802

803

804

805

806

807

808

809

810

811

812

813

814

815

816

817

818

(A) Representative bright-field image of 1-week old iNeurons differentiated from iPSCderived NPCs plated on a 16-electrode array of an MEA well. Spontaneous action potentials were recorded from the homozygous SYNGAP1 null (Homo\#4 and \#30) and control (WT\#6 and \#30) neurons. (B) Representative wave forms of spiking behavior from a single electrode for each Homo and WT neuronal culture. (C) Representative temporal raster plots of $\mathrm{KO}$ iNeurons (KO\#4 and \#38) and WT isogenic control iNeurons (WT\#6 and \#30) over 5-minuntes of continuous recording during culture week 3. (D-E) Cumulative plots of mean firing rates for all four clones individually (D) and grouped together by genotype (E), along a developmental timeline. (F-G) Cumulative plots of average number of bursts for individual clones $(\mathbf{F})$ and grouped together by genotype $(\mathbf{G})$. (H-I) Cumulative plots of average number of network bursts for all clone individually $(\mathbf{H})$ and grouped together by genotype (I). KO neurons display synaptic connections as early as week 3 of maturation compared to the WT controls. (Week 2 - Clonal analysis, $\mathrm{H}=9.331$, $\mathrm{p}=0.0096$, post-hoc comparisons $\mathrm{p}=0.0227$ for KO\#38 vs WT\#6, $p>0.999$ for WT\#6 vs WT\#30; $p=0.2697$ for WT\#6 vs KO\#4; $p>0.9999$ for KO\#4 vs KO\#38 and $p=0.999$ for WT\#30 vs KO\#4 and $p=0.3803$ for WT\#30 vs KO\#38; Genotype analysis, $U=6, p=0.0047$; Week $3-H=12.73 p<0.0001$, post-hoc comparisons $p=0.0140$ for KO\#4 vs WT\#6, $p=0.0227$ for KO\#4 vs WT\#30;Genotype analysis $U=0, p=0.0002$; Week 4 - Clonal analysis, $\mathrm{H}=12.29 \mathrm{p}=0.0001$, post-hoc comparisons $\mathrm{p}=0.01410$ for $\mathrm{KO \# 4}$ vs WT\#30; Genotype analysis, $U=0, p=0.0002$; Week 5 - Clonal analysis, $H=11.89 \mathrm{p}=0.0004$, post-hoc comparisons $p=0.0084$ for KO\#4 vs WT\#6; Genotype analysis, $U=2, p=0.0006$; Week $6-\mathrm{H}=9.088, \mathrm{p}=0.0117$; post-hoc comparisons $\mathrm{p}=0.0451$ for KO\#4 vs WT\#6; Genotype analysis, $\mathrm{U}=10, \mathrm{p}=0.0207$ ). $\mathrm{N}=4$ replicas for $\mathrm{WT} \# 6$, WT\#30, KO\#4 and $\mathrm{KO} \# 38 ; \mathrm{N}=8$ replicas from $\mathrm{WTH6+30}$ and $\mathrm{KO} \# 4+38$. For each clone, four replicates of iNeurons were plated and differentiated concurrently. Bar graph represents mean \pm SEM. ${ }^{*} p<0.05,{ }^{* *} p<0.01,{ }^{* * *} p<0.001$ and ${ }^{* * * *} p<0.0001$. 


\section{References}

Aceti M, Creson TK, Vaissiere T, Rojas C, Huang WC, Wang YX, Petralia RS, Page DT, Miller CA, Rumbaugh G (2015) Syngap1 haploinsufficiency damages a postnatal critical period of pyramidal cell structural maturation linked to cortical circuit assembly. Biological psychiatry 77:805-815.

Berryer MH et al. (2013) Mutations in SYNGAP1 cause intellectual disability, autism, and a specific form of epilepsy by inducing haploinsufficiency. Human mutation 34:385-394.

Carvill GL et al. (2013) Targeted resequencing in epileptic encephalopathies identifies de novo mutations in CHD2 and SYNGAP1. Nat Genet 45:825-830.

Charrier C, Joshi K, Coutinho-Budd J, Kim JE, Lambert N, de Marchena J, Jin WL, Vanderhaeghen P, Ghosh A, Sassa T, Polleux F (2012) Inhibition of SRGAP2 function by its human-specific paralogs induces neoteny during spine maturation. Cell 149:923935.

Clement JP, Ozkan ED, Aceti M, Miller CA, Rumbaugh G (2013) SYNGAP1 links the maturation rate of excitatory synapses to the duration of critical-period synaptic plasticity. J Neurosci 33:10447-10452.

Clement JP, Aceti M, Creson TK, Ozkan ED, Shi Y, Reish NJ, Almonte AG, Miller BH, Wiltgen BJ, Miller CA, Xu X, Rumbaugh G (2012) Pathogenic SYNGAP1 mutations impair cognitive development by disrupting maturation of dendritic spine synapses. Cell 151:709-723.

Deciphering Developmental Disorders S (2015) Large-scale discovery of novel genetic causes of developmental disorders. Nature 519:223-228.

Deciphering Developmental Disorders S (2017) Prevalence and architecture of de novo mutations in developmental disorders. Nature 542:433-438.

Fischer SKrnGF Multielectrode array recordings of human iPSC-derived neurons reveal differences in network activity depending on differentiation protocol and genome modification. Cellular Neuroscience Archive.

Gamache TR, Araki Y, Huganir RL (2020) Twenty Years of SynGAP Research: From Synapses to Cognition. J Neurosci 40:1596-1605.

Gou G, Roca-Fernandez A, Kilinc M, Serrano E, Reig-Viader R, Araki Y, Huganir RL, de Quintana-Schmidt C, Rumbaugh G, Bayés À (2020) SynGAP splice variants display heterogeneous spatio-temporal expression and subcellular distribution in the developing mammalian brain. Journal of Neurochemistry n/a.

Hamdan FF, Daoud H, Piton A, Gauthier J, Dobrzeniecka S, Krebs MO, Joober R, Lacaille JC, Nadeau A, Milunsky JM, Wang Z, Carmant L, Mottron L, Beauchamp MH, Rouleau GA, Michaud JL (2011) De novo SYNGAP1 mutations in nonsyndromic intellectual disability and autism. Biological psychiatry 69:898-901.

Hamdan FF et al. (2009) Mutations in SYNGAP1 in autosomal nonsyndromic mental retardation. The New England journal of medicine 360:599-605.

Jiang X, Chen J, Bajić A, Zhang C, Song X, Carroll SL, Cai Z-L, Tang M, Xue M, Cheng N, Schaaf CP, Li F, MacKenzie KR, Ferreon ACM, Xia F, Wang MC, Maletić-Savatić M, Wang J (2017) Quantitative real-time imaging of glutathione. Nature communications 8:16087.

Jimenez-Gomez A, Niu S, Andujar-Perez F, McQuade EA, Balasa A, Huss D, Coorg R, Quach M, Vinson S, Risen S, Holder JL, Jr. (2019) Phenotypic characterization of individuals with SYNGAP1 pathogenic variants reveals a potential correlation between posterior dominant rhythm and developmental progression. J Neurodev Disord 11:18.

Kilinc M, Creson T, Rojas C, Aceti M, Ellegood J, Vaissiere T, Lerch JP, Rumbaugh G (2018) Species-conserved SYNGAP1 phenotypes associated with neurodevelopmental disorders. Mol Cell Neurosci 91:140-150. 
870

871

872

873

874

875

876

877

878

879

880

881

882

883

884

885

886

887

888

889

890

891

892

893

894

895

896

897

898

899

900

901

902

903

904

905

906

907

908

909

910

911

912

913

914

915

916

917

918

919

920

Kim JH, Lee HK, Takamiya K, Huganir RL (2003) The role of synaptic GTPase-activating protein in neuronal development and synaptic plasticity. J Neurosci 23:1119-1124.

Komiyama NH, Watabe AM, Carlisle HJ, Porter K, Charlesworth P, Monti J, Strathdee DJ, O'Carroll CM, Martin SJ, Morris RG, O'Dell TJ, Grant SG (2002) SynGAP regulates ERK/MAPK signaling, synaptic plasticity, and learning in the complex with postsynaptic density 95 and NMDA receptor. J Neurosci 22:9721-9732.

Lek $M$ et al. (2016) Analysis of protein-coding genetic variation in 60,706 humans. Nature 536:285-291.

McMahon AC, Barnett MW, O'Leary TS, Stoney PN, Collins MO, Papadia S, Choudhary JS, Komiyama NH, Grant SG, Hardingham GE, Wyllie DJ, Kind PC (2012) SynGAP isoforms exert opposing effects on synaptic strength. Nature communications 3:900.

Michaelson SD, Ozkan ED, Aceti M, Maity S, Llamosas N, Weldon M, Mizrachi E, Vaissiere T, Gaffield MA, Christie JM, Holder JL, Jr., Miller CA, Rumbaugh G (2018) SYNGAP1 heterozygosity disrupts sensory processing by reducing touch-related activity within somatosensory cortex circuits. Nature neuroscience 21:1-13.

Mignot C et al. (2016) Genetic and neurodevelopmental spectrum of SYNGAP1-associated intellectual disability and epilepsy. J Med Genet 53:511-522.

Nehme R et al. (2018) Combining NGN2 Programming with Developmental Patterning Generates Human Excitatory Neurons with NMDAR-Mediated Synaptic Transmission. Cell Rep 23:2509-2523.

O'Roak BJ, Stessman HA, Boyle EA, Witherspoon KT, Martin B, Lee C, Vives L, Baker C, Hiatt JB, Nickerson DA, Bernier R, Shendure J, Eichler EE (2014) Recurrent de novo mutations implicate novel genes underlying simplex autism risk. Nature communications 5:5595.

Ozkan ED, Creson TK, Kramar EA, Rojas C, Seese RR, Babyan AH, Shi Y, Lucero R, Xu X, Noebels JL, Miller CA, Lynch G, Rumbaugh G (2014) Reduced cognition in Syngap1 mutants is caused by isolated damage within developing forebrain excitatory neurons. Neuron 82:1317-1333.

Parker MJ, Fryer AE, Shears DJ, Lachlan KL, McKee SA, Magee AC, Mohammed S, Vasudevan PC, Park SM, Benoit V, Lederer D, Maystadt I, Study D, FitzPatrick DR (2015) De novo, heterozygous, loss-of-function mutations in SYNGAP1 cause a syndromic form of intellectual disability. Am J Med Genet A 167A:2231-2237.

Petanjek Z, Judas M, Simic G, Rasin MR, Uylings HB, Rakic P, Kostovic I (2011) Extraordinary neoteny of synaptic spines in the human prefrontal cortex. Proceedings of the National Academy of Sciences of the United States of America 108:13281-13286.

Rauch A et al. (2012) Range of genetic mutations associated with severe non-syndromic sporadic intellectual disability: an exome sequencing study. Lancet 380:1674-1682.

Rumbaugh G, Adams JP, Kim JH, Huganir RL (2006) SynGAP regulates synaptic strength and mitogen-activated protein kinases in cultured neurons. Proceedings of the National Academy of Sciences of the United States of America 103:4344-4351.

Satterstrom FK et al. (2020) Large-Scale Exome Sequencing Study Implicates Both Developmental and Functional Changes in the Neurobiology of Autism. Cell 180:568584 e523.

Sridharan B, Hubbs C, Llamosas N, Kilinc M, Singhera FU, Willems E, Piper DR, Scampavia L, Rumbaugh G, Spicer TP (2019) A Simple Procedure for Creating Scalable Phenotypic Screening Assays in Human Neurons. Scientific Reports 9:9000.

Suresh J, Radojicic M, Pesce LL, Bhansali A, Wang J, Tryba AK, Marks JD, van Drongelen W (2016) Network burst activity in hippocampal neuronal cultures: the role of synaptic and intrinsic currents. J Neurophysiol 115:3073-3089.

Vlaskamp DRM et al. (2019) SYNGAP1 encephalopathy: A distinctive generalized developmental and epileptic encephalopathy. Neurology 92:e96-e107. 
bioRxiv preprint doi: https://doi.org/10.1101/2020.06.01.127613; this version posted June 3, 2020. The copyright holder for this preprint (which

was not certified by peer review) is the author/funder, who has granted bioRxiv a license to display the preprint in perpetuity. It is made available under aCC-BY-NC-ND 4.0 International license.

921 von Stulpnagel C, Funke C, Haberl C, Hortnagel K, Jungling J, Weber YG, Staudt M, Kluger G (2015) SYNGAP1 Mutation in Focal and Generalized Epilepsy: A Literature Overview and A Case Report with Special Aspects of the EEG. Neuropediatrics 46:287-291. on SYNGAP1-related brain disorders: a stakeholder meeting of families, researchers, clinicians, and regulators. J Neurodev Disord 10:6.

Zhang Y, Pak C, Han Y, Ahlenius H, Zhang Z, Chanda S, Marro S, Patzke C, Acuna C, Covy J, Xu W, Yang N, Danko T, Chen L, Wernig M, Sudhof TC (2013) Rapid single-step induction of functional neurons from human pluripotent stem cells. Neuron 78:785-798. 
Table 1. Indels present in the clonal SYNGAP1 iPSC lines (relative to starting material)

WT \#6

\begin{tabular}{|l|l|l|l|l|l|l|}
\hline $\begin{array}{l}\text { Allele } \\
\text { freq } \\
\text { of } \\
\text { sampl } \\
\text { e }\end{array}$ & $\begin{array}{l}\text { Allele } \\
\text { freq in } \\
\text { Cas9 }\end{array}$ & $\begin{array}{l}\text { Covera } \\
\text { ge }\end{array}$ & $\begin{array}{l}\text { Chro } \\
\mathrm{m}\end{array}$ & Position & Cas9 sequence & $\begin{array}{l}\text { Sample } \\
\text { sequen } \\
\text { ce }\end{array}$ \\
\hline 0.5 & $\begin{array}{l}0.00011 \\
96\end{array}$ & 31 & 10 & $\begin{array}{l}1935249 \\
1\end{array}$ & AGT & A \\
\hline 0.5 & 0.032 & 28 & 17 & $\begin{array}{l}8099450 \\
7\end{array}$ & $\begin{array}{l}\text { TGCCTGGCGCTCAGTAGCGTGGCCAGGGCTCCCAGTGTGGG } \\
\text { CTCGGTGAC }\end{array}$ & T \\
\hline 0.5 & 0.068 & 34 & 20 & $\begin{array}{l}2493069 \\
4\end{array}$ & TC & T \\
\hline 0.529 & 0.00011 & 29 & 5 & $\begin{array}{l}1765083 \\
92\end{array}$ & C & CA \\
\hline 0.554 & 0.252 & 44 & 8 & $\begin{array}{l}5842620 \\
2\end{array}$ & CT & C \\
\hline 0.556 & 0.065 & 33 & 8 & $\begin{array}{l}1414603 \\
62\end{array}$ & CT & C \\
\hline 0.641 & 0.222 & 41 & 10 & $\begin{array}{l}1017984 \\
08\end{array}$ & CT & C \\
\hline
\end{tabular}

WT \#30

\begin{tabular}{|l|l|l|l|l|l|l|l|}
\hline $\begin{array}{l}\text { Allele freq of } \\
\text { sample }\end{array}$ & $\begin{array}{l}\text { Allele freq in } \\
\text { Cas9 }\end{array}$ & Coverage & Chrom & Position & & Cas9 sequence & Sample sequence \\
\hline 0.5 & 0.0001432 & 34 & 21 & 10483254 & $\cdot$ & T & TA \\
\hline 0.5 & 0.0005416 & 24 & 8 & 8066187 & $\cdot$ & T & TG \\
\hline 0.502 & 0.148 & 34 & 14 & 69119490 & $\cdot$ & T & TA \\
\hline 0.502 & 0.019 & 722 & 19 & 54456134 & $\cdot$ & TCAC & T \\
\hline 0.507 & 0.029 & 22 & 6 & 4121693 & $\cdot$ & G & GT \\
\hline 0.509 & 0.045 & 205 & 18 & 14534945 & $\cdot$ & T & TA \\
\hline 0.557 & 0.026 & 31 & 5 & 93682503 & $\cdot$ & G & GT \\
\hline 0.585 & 0.02 & 27 & 5 & 142893941 & $\cdot$ & G & GT \\
\hline 0.643 & $2.76 \mathrm{E}-05$ & 27 & 9 & 91711724 & $\cdot$ & TAA & T \\
\hline 0.643 & $2.76 \mathrm{E}-05$ & 27 & 9 & 91711733 & $\cdot$ & G & GGTA \\
\hline
\end{tabular}


bioRxiv preprint doi: https://doi.org/10.1101/2020.06.01.127613; this version posted June 3, 2020. The copyright holder for this preprint (which was not certified by peer review) is the author/funder, who has granted bioRxiv a license to display the preprint in perpetuity. It is made available under ACC-BY-NC-ND 4.0 International license.

Table 1. (continued)

KO \#4

\begin{tabular}{|l|l|l|l|l|l|l|l|}
\hline $\begin{array}{l}\text { Allele freq of } \\
\text { sample }\end{array}$ & Allele freq in Cas9 & Coverage & Chrom & Position & & $\begin{array}{l}\text { Cas9 } \\
\text { sequence }\end{array}$ & Sample sequence \\
\hline 0.5 & 0.295 & 49 & 9 & 78300710 & $\cdot$ & C & CTT \\
\hline 0.5 & 0.119 & 29 & 9 & 91711722 & $\cdot$ & TGTAA & T \\
\hline 0.503 & 0.001171 & 23 & 13 & 108228871 & $\cdot$ & TA & T \\
\hline 0.505 & 0.061 & 33 & 18 & 23795721 & $\cdot$ & TA & T \\
\hline 0.553 & 0.116 & 25 & 22 & 31711776 & $\cdot$ & C & CA \\
\hline 0.556 & 0.192 & 65 & 21 & 43757986 & $\cdot$ & CT & C \\
\hline 0.6 & 0.247 & 45 & $X$ & 12817468 & $\cdot$ & T & TA \\
\hline 0.614 & 0.19 & 51 & 17 & 3814729 & $\cdot$ & C & CT \\
\hline 0.616 & 0.093 & 23 & 19 & 41837694 & $\cdot$ & C & CA \\
\hline 0.616 & 0.07 & 27 & 1 & 6581663 & $\cdot$ & CA & C \\
\hline 0.64 & 0.087 & 31 & 6 & 34819141 & $\cdot$ & C & CT \\
\hline 0.721 & 0.356 & 74 & 11 & 30873342 & $\cdot$ & TAC & T \\
\hline 0.781 & 0.275 & 38 & 2 & 96827265 & $\cdot$ & CT & C \\
\hline 0.972 & 0.022 & 315 & 6 & 33435551 & $\cdot$ & CG & C \\
\hline
\end{tabular}

\section{KO \#38}

\begin{tabular}{|l|l|l|l|l|l|l|}
\hline $\begin{array}{l}\text { Allele freq of } \\
\text { sample }\end{array}$ & $\begin{array}{l}\text { Allele freq in } \\
\text { Cas9 }\end{array}$ & Coverage & Chrom & Position & Cas9 sequence & $\begin{array}{l}\text { Sample } \\
\text { sequence }\end{array}$ \\
\hline 0.5 & 0.079 & 28 & 11 & 19155581 & C & CA \\
\hline 0.5 & 0.126 & 27 & 7 & 23505164 & TA & T \\
\hline 0.539 & 0.068 & 28 & 12 & 69284463 & GT & G \\
\hline 0.545 & 0.202 & 42 & 16 & 11156369 & C & CA \\
\hline 0.554 & 0.127 & 45 & 18 & 59824581 & A & AT \\
\hline 0.573 & 0.109 & 36 & 19 & 17988154 & C & CA \\
\hline 0.578 & 0.111 & 28 & 5 & 138505807 & T & TA \\
\hline 0.584 & 0.19 & 56 & 1 & 27552400 & CT & CAAA \\
\hline 0.585 & 0.216 & 41 & 6 & 34219990 & C & CAAAAA \\
\hline 0.616 & 0.043 & 23 & 7 & 101627137 & C & AT \\
\hline 0.666 & 0.093 & 26 & 7 & 48844654 & A & GCAGTGTT \\
\hline 0.778 & 0.021 & 39 & 9 & 130349951 & G & T \\
\hline 0.933 & 0.00798 & 292 & 6 & 33435544 & TGCCTGTCG & \\
\hline
\end{tabular}

